\title{
A WALL CROSSING FORMULA OF DONALDSON-THOMAS INVARIANTS WITHOUT CHERN-SIMONS FUNCTIONAL*
}

\author{
YOUNG-HOON KIEM ${ }^{\dagger}$ AND JUN LI ${ }^{\ddagger}$
}

Abstract. We prove a wall crossing formula of Donaldson-Thomas type invariants without Chern-Simons functionals.

Key words. Donaldson-Thomas invariant, wall crossing, intrinsic blowup.

AMS subject classifications. 14N35, 14D20.

1. Introduction. A wall crossing formula refers to the change of invariants of moduli spaces when they undergo birational transformations, like the variation of moduli spaces of stable sheaves when the stability condition changes. Wall crossing formulas were investigated extensively for the Donaldson polynomial invariants of surfaces in the 1990s by several groups, including Friedman-Qin [6], Ellingsurd-Göttsche [5] and Matsuki-Wentworth [17]. At that time, the notion of virtual cycle had not been discovered and wall crossing formulas were worked out largely for smooth moduli spaces. Wall crossing formulas involving virtual cycles were taken up by T. Mochizuki [18] in his work on higher rank Donaldson polynomial invariants of surfaces. All these approaches use Geometric Invariant Theory (or GIT for short) flips discovered by Dolgachev-Hu and Thaddeus $[4,23]$, relying on that the moduli spaces of sheaves are constructed using GIT [7, 16, 21].

For a Calabi-Yau three-fold $S$, the moduli space of stable sheaves with fixed Chern classes is equipped with a symmetric obstruction theory; the degree of its virtual fundamental class defines the Donaldson-Thomas invariant of $S$. In [10, Thm. 5.11], using the existence of local Chern-Simons functionals, Joyce-Song prove a wall crossing formula in case the stability crosses a wall (cf. Definition 2.1). Here a local Chern-Simons functional refers to a function $f$ defined on a smooth $Y$ such that the vanishing of $d f$ defines the germ of the moduli space and its symmetric obstruction theory. By using Hall algebras and breaking the moduli spaces into pieces, they define generalized Donaldson-Thomas invariants for arbitrary Chern classes and find formulas comparing them.

Recently, the study of moduli of stable sheaves has been extended to moduli of stable objects in the derived category $D^{b}(\mathrm{Coh} S)$ of bounded complexes of coherent sheaves. In general, the study of moduli of complexes of coherent sheaves or objects in the derived category cannot be reduced to the study of sheaves, and the existence of local Chern-Simons functionals is not known. Therefore, in order to extend the wall crossing formulas to moduli of stable objects in the derived category, a new method is required.

In this paper we develop a new method for wall crossing formulas of DonaldsonThomas type invariants without relying on Chern-Simons functionals. Our method is not motivic and does not use Behrend's function. Instead we perform a $\mathbb{C}^{*}$-intrinsic

\footnotetext{
*Received February 11, 2011; accepted for publication March 6, 2012

${ }^{\dagger}$ Department of Mathematics and Research Institute of Mathematics, Seoul National University, Seoul 151-747, Korea (kiem@math.snu.ac.kr). Young-Hoon Kiem was partially supported by NRF 2011-0027969.

$\ddagger$ Department of Mathematics, Stanford University, Stanford, USA (jli@math.stanford.edu). Jun Li was partially supported by NSF grant NSF-0601002.
} 
blow-up to resolve the issue of infinite stabilizers, construct an auxiliary space (called the master space) that captures the wall crossing phenomenon, and apply the virtual localization formula [8] and our reduction technique [11].

We now outline our results. We let $\mathcal{M}=\left[X / \mathbb{C}^{*}\right]$ be the quotient of a separated $\mathbb{C}^{*}$ equivariant Deligne-Mumford (or DM for short) stack $X$ equipped with an equivariant symmetric obstruction theory (Definition 2.11). Suppose $\mathcal{M}$ contains two open dense substacks $M_{ \pm}=\left[X_{ \pm} / \mathbb{C}^{*}\right]$ that are separated proper DM-stacks. The equivariant symmetric obstruction theory of $X$ induces symmetric obstruction theories of $M_{ \pm}$. By $[1,13]$, the symmetric obstruction theories provide their respective (dimension 0) virtual cycles $\left[M_{ \pm}\right]^{\mathrm{vir}}$. The wall crossing formula measures the difference $\operatorname{deg}\left[M_{+}\right]^{\mathrm{vir}}-$ $\operatorname{deg}\left[M_{-}\right]^{\text {vir }}$.

Taking the $\mathbb{C}^{*}$-fixed part of the symmetric obstruction theory along the fixed point locus $X^{\mathbb{C}^{*}}$, we have an induced symmetric obstruction theory on $X^{\mathbb{C}^{*}}$. We let its zero dimensional virtual fundamental class be

$$
\left[X^{\mathbb{C}^{*}}\right]^{\text {vir }}=\sum_{k=1}^{r} a_{k}\left[p_{k}\right] ; \quad p_{k} \in X^{\mathbb{C}^{*}}, a_{k} \in \mathbb{Q} .
$$

TheOrem 1.1. Suppose $M_{ \pm} \subset \mathcal{M}$ is a simple flip (Definition 2.2) and $\mathcal{M}=$ $\left[X / \mathbb{C}^{*}\right]$ has a symmetric obstruction theory (Definition 2.11). Suppose further that $X$ can be embedded $\mathbb{C}^{*}$-equivariantly into a smooth DM-stack ([8, Appendix C]). Then

$$
\operatorname{deg}\left[M_{+}\right]^{\mathrm{vir}}-\operatorname{deg}\left[M_{-}\right]^{\mathrm{vir}}=\sum_{k} a_{k} \cdot \lambda_{k} ; \quad \lambda_{k}=(-1)^{n_{k}-1} \sum_{j} n_{k, j} / j,
$$

where $n_{k, j}$ is the dimension of the weight $j$ part of the Zariski tangent space $T_{X, p_{k}}$.

In case $\lambda_{k}=\lambda$ is independent of $k$, then

$$
\operatorname{deg}\left[M_{+}\right]^{\text {vir }}-\operatorname{deg}\left[M_{-}\right]^{\text {vir }}=\lambda \cdot \operatorname{deg}\left[X^{\mathbb{C}^{*}}\right]^{\text {vir }} .
$$

We comment that the proof does not require the existence of local Chern-Simons functionals.

Our proof goes as follows. We first construct a $\mathbb{C}^{*}$-intrinsic blow-up $\bar{X}$ of $X$ along $X^{\mathbb{C}^{*}}$ and show that the quotient $\left[\bar{X} / \mathbb{C}^{*}\right]$ has an induced perfect obstruction theory. We then construct a master space $\bar{Z}$ for $\bar{X}$, analogous to that in [23] and apply the localization by cosection technique [11] to construct a reduced virtual fundamental class of $\bar{Z}$. Applying the virtual localization formula of [8] to $\bar{Z}$, we obtain Theorem 1.1 .

It is worthwhile to comment on the assumptions of Theorem 1.1. As shown below, the existence of $X$ is assured in quite a general setting. But embedding $X \mathbb{C}^{*}$ equivariantly into a smooth DM-stack seems restrictive. In two occasions of the proof we use this assumption; one is constructing the obstruction theory of the $\mathbb{C}^{*}$-intrinsic blow-up, the other is applying Graber-Pandharipande's virtual $\mathbb{C}^{*}$-localization theorem.

Here is what we plan to do to remove this technical assumption in the near future. Firstly, we intend to develop a local obstruction theory that consists of an étale atlas $U_{\alpha} \rightarrow X$, obstruction theories $\eta_{\alpha}: F_{\alpha}^{\bullet} \rightarrow \mathbb{L}_{U_{\alpha}}$, and compatibility of the obstruction theories over $U_{\alpha \beta}=U_{\alpha} \times_{X} U_{\beta}$. Such a theory is known to the experts, and the usual tools on virtual cycles apply. This seems necessary since it is hard to construct a 
universal family of the moduli stack of derived category objects. Secondly, we intend to prove the $\mathbb{C}^{*}$-localization theorem in the setting of local obstruction theory. This seems to require more efforts, but should be achievable in the near future.

In light of this, in this paper we also prove some technical results aimed at proving the following generalization.

CONJECTURE 1.2. Theorem 1.1 holds true without assuming the existence of a $\mathbb{C}^{*}$-equivariant embedding of $X$ into a smooth DM-stack.

We now comment on how Theorem 1.1 applies toward a wall crossing formula of Donaldson-Thomas invariants of moduli of derived category objects over a Calabi-Yau three-fold $S$. Let $\tau_{ \pm}$be two stability conditions crossing a simple wall $\tau_{0}$ (Definition 2.1 ); let $\mathcal{M}$ be the moduli stack of $\tau_{0}$-semistable objects. We construct a separated DM-stack $X$ parameterizing pairs $(E, \sigma)$, where $E \in \mathcal{M}$ and $\sigma: L(E) \stackrel{\cong}{\longrightarrow}$ for some 1-dimensional vector space $L(E)$ associated to $E$. Scaling $\sigma$ with elements in $\mathbb{C}^{*}$ makes $X$ a $\mathbb{C}^{*}$-equivariant stack. Then $\mathcal{M}=\left[X / \mathbb{C}^{*}\right]$, while the moduli stacks of $\tau_{ \pm}$-stable objects are open substacks $M_{ \pm}=\left[X_{ \pm} / \mathbb{C}^{*}\right]$ for two open substacks $X_{ \pm} \subset X$ (cf. Proposition 2.9). Because Conjecture 1.2 is not proved yet, we suppose that $X$ equivariantly embeds into a smooth DM-stack. This condition is always satisfied when $\mathcal{M}$ is constructed as a GIT quotient. In particular, our results apply directly to the moduli of sheaves or stable pairs. We show in Lemma 2.13 that $X$ has a $\mathbb{C}^{*}$ equivariant symmetric obstruction theory. It is now immediate to deduce the following wall crossing formula from Theorem 1.1.

COROllary 1.3. Let $\tau_{ \pm}$be two stability conditions crossing a simple wall $\tau_{0}$ (Definition 2.1); let $\mathcal{M}$ be the moduli stack of $\tau_{0}$-semistable objects $D^{b}(\mathrm{Coh} S)$ with the same fixed Chern classes, and let $M_{ \pm} \subset \mathcal{M}$ be the moduli stacks of $\tau_{ \pm}$-stable objects. Suppose $M_{ \pm} \subset \mathcal{M}$ is a simple wall crossing of each other (cf. Definition 2.1). Suppose the DM stack $X$ constructed in Proposition 2.9 embeds $\mathbb{C}^{*}$-equivariantly in a smooth DM stack. Suppose further that $\operatorname{Ext}^{\leq-1}(E, E)=0$ for all $E \in \mathcal{M}$. Then

$$
\operatorname{deg}\left[M_{+}\right]^{\text {vir }}-\operatorname{deg}\left[M_{-}\right]^{\text {vir }}=(-1)^{\chi\left(E_{1}, E_{2}\right)-1} \cdot \chi\left(E_{1}, E_{2}\right) \cdot \operatorname{deg}\left[M_{1}\right]^{\text {vir }} \cdot \operatorname{deg}\left[M_{2}\right]^{\text {vir }},
$$

where $E_{i} \in M_{i}, M_{i}$ are the moduli stacks specified in Definition 2.1 and $\chi\left(E_{1}, E_{2}\right)=$ $\sum(-1)^{i} \operatorname{dim} \operatorname{Ext}^{i}\left(E_{1}, E_{2}\right)$.

In $\S 2$, we show that a simple wall crossing $M_{ \pm} \subset \mathcal{M}$ in $D^{b}(\operatorname{Coh} S)$ is described as a simple flip $\left[X_{ \pm} / \mathbb{C}^{*}\right] \subset\left[X / \mathbb{C}^{*}\right]$. In $\S 3$, we construct an intrinsic blow-up of $\mathbb{C}^{*}$ quotients and study the induced perfect obstruction theory. In $\S 4$, we construct the master space and in $\S 5$, we apply the virtual localization formula of [8] to obtain Theorem 1.1 and Corollary 1.3 by careful local calculations. In the Appendix, we discuss an analogous wall crossing result for non-symmetric obstruction theories.

2. Donaldson-Thomas invariants and wall crossing. In this section, we explain how a simple wall crossing (Definition 2.1) can be described by a simple flip (Definition 2.2) in a global $\mathbb{C}^{*}$ quotient equipped with an equivariant obstruction theory. In later sections, we will work out a wall crossing formula for simple flips.

2.1. Simple wall crossing. In this paper, we fix a smooth projective CalabiYau three-fold $S$. Let $\tau_{0}$ be a stability condition in some abelian subcategory $\mathcal{A}_{0}$ of the derived category $D^{b}(\operatorname{Coh}(S))$ of bounded complexes of coherent sheaves. Let $\mathcal{M}$ be the moduli stack of $\tau_{0}$-semistable objects, with fixed Chern classes. By tensoring with some power of $\mathcal{O}_{S}(1)$ if necessary, we may assume $\chi(E) \neq 0$ for $E \in \mathcal{M}$. 
Let $\tau_{ \pm}$be nearby stability conditions of $\tau_{0}$ and let $M_{ \pm}$be the moduli stack of $\tau_{ \pm}$-stable objects. In this paper, we consider the following case of simple wall crossing.

Definition 2.1. We say $M_{+}$is obtained from $M_{-}$by a simple wall crossing in $\mathcal{M}$ if the following conditions are satisfied:

1. Strictly $\tau_{0}$-semistable objects $E$ (i.e. semistable but not stable) in $\mathcal{M}$ have $\operatorname{gr}(E)=E_{1} \oplus E_{2}$ with $E_{1} \in M_{1}$ and $E_{2} \in M_{2}$, where $M_{i}$ are the moduli stacks of $\tau_{0}$-stable objects of Chern classes equal to $c\left(E_{i}\right)$. Further, both $M_{1}$ and $M_{2}$ are proper separated DM-stacks;

2. the factors $E_{i} \in M_{i}$ in (1) satisfy $\chi\left(E_{1}\right)=\chi\left(E_{2}\right)$; there is a $B \in K(S)$ such that $\chi\left(E_{1} \otimes B\right) \neq \chi\left(E_{2} \otimes B\right)$;

3. $\tau_{+}$-stable (resp. $\tau_{-}$-stable) objects are either $\tau_{0}$-stable or non-split extensions of $E_{2}$ by $E_{1}$ (resp. $E_{1}$ by $E_{2}$ ) for some strictly $\tau_{0}$-semistable objects $E_{1} \oplus E_{2}$ with $E_{1} \in M_{1}$ and $E_{2} \in M_{2}$

4. $M_{ \pm}$are proper separated DM-stacks.

It is immediate that $M_{ \pm}$are open substacks of $\mathcal{M}$. The wall crossing formula compares the degrees of the virtual fundamental classes of $M_{+}$and $M_{-}$.

We remark that part of the Definition requires that all elements in $M_{1}$ (resp. $M_{2}$ ) have identical Chern classes $c\left(E_{1}\right)$ (resp. $c\left(E_{2}\right)$ ). The case where the wall crossing occurs at different $c\left(E_{1}\right)$ and $c\left(E_{2}\right)$ can be treated the same way since then $M_{1}$ and $M_{2}$ splits to pairs $\left(M_{1, j}, M_{2, j}\right)$, where elements in $M_{1, j}$ and $M_{2, j}$ have identical Chern classes.

2.2. Simple flip. In this subsection, we introduce our geometric set-up for simple wall crossing. Let $X$ be a separated Deligne-Mumford stack of finite type acted on by $\mathbb{C}^{*}$. Let $\mathcal{M}=\left[X / \mathbb{C}^{*}\right]$ be the quotient stack.

Let $X^{\mathbb{C}^{*}}$ denote the fixed point locus in $X$. We let $X_{0} \subset X-X^{\mathbb{C}^{*}}$ be the open substack of $x \in X$ so that the orbit $\mathbb{C}^{*} \cdot x$ is 1 -dimensional and closed in $X$, and let

$$
\Sigma_{ \pm}^{\circ}=\left\{x \in X-X_{0} \cup X^{\mathbb{C}^{*}} \mid \lim _{\sigma \rightarrow 0} \sigma^{ \pm 1} \cdot x \in X^{\mathbb{C}^{*}}\right\}
$$

We then form

$$
\Sigma_{ \pm}=\Sigma_{ \pm}^{\circ} \cup X^{\mathbb{C}^{*}}
$$

which are $\mathbb{C}^{*}$-invariant, and let

$$
X_{ \pm}=X-\Sigma_{\mp} \subset X, \quad M_{ \pm}=\left[X_{ \pm} / \mathbb{C}^{*}\right] \subset \mathcal{M}=\left[X / \mathbb{C}^{*}\right]
$$

Definition 2.2. We say that the pair $M_{ \pm}=\left[X_{ \pm} / \mathbb{C}^{*}\right] \subset \mathcal{M}$ is a simple flip if the following hold:

(i) The fixed locus $X^{\mathbb{C}^{*}}$ is a proper DM-stack;

(ii) $\Sigma_{ \pm}$are closed in $X$;

(iii) both $M_{ \pm}=\left[X_{ \pm} / \mathbb{C}^{*}\right]$ are proper separated DM-stacks;

(iv) the pair $X_{ \pm} \subset X$ satisfies the Simpleness Condition stated below.

We first fix our convention on discrete valuation rings and their finite extensions. Let $R$ be a discrete valuation ring. We always denote by $K$ its field of fractions; $\xi$ and $\xi_{0}$ be the generic and closed point of $\operatorname{Spec} R$. When $\widetilde{R} \supset R$ is a finite extension, we denote by $\tilde{K}, \tilde{\xi}$ and $\tilde{\xi}_{0}$ the corresponding field of fractions, the generic point and the closed point. We let $\zeta$ and $\tilde{\zeta}$ be uniformizing parameters of $R$ and $\tilde{R}$, respectively. We 
denote by $\imath(\tilde{R})$ the ramification index of $\tilde{R} \supset R$; namely, $\zeta=\tilde{\gamma} \cdot \tilde{\zeta}^{\imath(\tilde{R})}$ for an intertible $\tilde{\gamma} \in \tilde{R}$. In case $f: \operatorname{Spec} R \rightarrow X$ is a morphism, we denote by $\tilde{f}:$ Spec $\tilde{R} \rightarrow X$ the composite Spec $\tilde{R} \rightarrow \operatorname{Spec} R \rightarrow X$. Given $g: \operatorname{Spec} K \rightarrow \mathbb{C}^{*}$, we denote by $g \cdot f$ the composition

$$
g \cdot f: \operatorname{Spec} K \stackrel{\Delta}{\longrightarrow} \operatorname{Spec} K \times \operatorname{Spec} K \hookrightarrow \operatorname{Spec} K \times \operatorname{Spec} R \stackrel{(g, f)}{\longrightarrow} \mathbb{C}^{*} \times X \longrightarrow X,
$$

where the first arrow is the diagonal embedding and the last arrow is the $\mathbb{C}^{*}$-action on $X$.

Simpleness CONDITION 2.3. Let $R$ be a discrete valuation ring over $\mathbb{C}$. Let $f_{+}: \operatorname{Spec} R \rightarrow X$; let $g: \operatorname{Spec} K \rightarrow \mathbb{C}^{*}$ be of the form $g^{*}(t)=\alpha \cdot \zeta^{a}$ for an invertible $\alpha \in R$ and $a>0$. Then the following hold:

(a) Suppose $f_{+}(\xi) \in X_{0}, f_{+}\left(\xi_{0}\right) \in \Sigma_{+}^{\circ}$, and $g \cdot f_{+}$: Spec $K \rightarrow X_{0}$ extends to $f_{-}: \operatorname{Spec} R \rightarrow X$ so that $f_{-}\left(\xi_{0}\right) \in \Sigma_{-}^{\circ}$. Then there is a finite extension $\tilde{R} \supset R$ such that for any invertible $\tilde{\beta} \in \tilde{R}$ and any integer $0<b<\imath(\tilde{R}) \cdot a$, the morphism $\tilde{h}:$ Spec $\tilde{K} \rightarrow \mathbb{C}^{*}$ of the form $\tilde{h}^{*}(t)=\tilde{\beta} \cdot \tilde{\zeta}^{b}$ has the property that $\tilde{h} \cdot \tilde{f}_{+}: \operatorname{Spec} \tilde{K} \rightarrow X$ extends to a morphism $\left(\tilde{h} \cdot \tilde{f}_{+}\right)^{\#}: \operatorname{Spec} \tilde{R} \rightarrow X$ with $\left(\tilde{h} \cdot \tilde{f}_{+}\right)^{\#}\left(\tilde{\xi}_{0}\right) \in X^{\mathbb{C}^{*}}$.

(b) Suppose $f_{+}:$Spec $R \rightarrow \Sigma_{+}^{\circ}$. Then possibly after a finite extension $\tilde{R} \supset R$, $\tilde{g} \cdot \tilde{f}_{+}: \operatorname{Spec} \tilde{K} \rightarrow \Sigma_{+}^{\circ}$ extends to a morphism $\left(\tilde{g} \cdot \tilde{f}_{+}\right)^{\#}: \operatorname{Spec} \tilde{R} \rightarrow \Sigma_{+}$such that $\left(\tilde{g} \cdot \tilde{f}_{+}\right) \#\left(\tilde{\xi}_{0}\right) \in X^{\mathbb{C}^{*}}$.

(c) Both (1) and (2) hold with "+ " replaced by "-", $a>0$ replaced by $a<0$, and $0<b<\imath(\tilde{R}) \cdot a$ replaced by $\imath(\tilde{R}) \cdot a<b<0$.

EXAmPLE 2.4. Let $V_{+}, V_{-}, V_{0}$ be vector spaces on which $\mathbb{C}^{*}$ acts with weights 1 , -1 , and 0 respectively. Let $V=V_{+} \times V_{0} \times V_{-}$and $X=\mathbb{P} V-\left(\mathbb{P} V_{+} \cup \mathbb{P} V_{-}\right)$. Then $\Sigma_{ \pm}=V_{ \pm} \otimes \mathcal{O}_{\mathbb{P} V_{0}}(1)$ and $X_{ \pm}=\mathbb{P} V-\mathbb{P}\left(V_{0} \times V_{\mp}\right)$. Hence $M_{+}=\mathbb{P} V_{+} \times \mathbb{P}\left(V_{0} \times V_{-}\right)$and $M_{-}=\mathbb{P} V_{-} \times \mathbb{P}\left(V_{0} \times V_{+}\right)$.

Suppose in addition that $M_{ \pm}$have symmetric obstruction theories. Since $M_{ \pm}$are smooth, their virtual cycles $\left[M_{ \pm}\right]^{\text {vir }}$ are equal to the Euler classes $e\left(\Omega_{M_{+}}\right)$. Therefore the wall crossing formula is

$$
\operatorname{deg}\left[M_{+}\right]^{\mathrm{vir}}-\operatorname{deg}\left[M_{-}\right]^{\mathrm{vir}}=(-1)^{n_{+}-n_{-}-1}\left(n_{+}-n_{-}\right) \cdot \operatorname{deg}\left[X^{\mathbb{C}^{*}}\right]^{\mathrm{vir}}
$$

where $n_{ \pm}=\operatorname{dim} V_{ \pm}$is the dimension of the \pm 1 -weight space in the normal space to $X^{\mathbb{C}^{*}}$ in $X$.

EXAMPLE 2.5. ([22]) More generally, suppose there is a $\mathbb{C}^{*}$-equivariant separated proper scheme $W$ such that

$$
X=W-\left\{x \in W \mid \lim _{t \rightarrow \infty} t \cdot x \in F^{+}\right\}-\left\{x \in W \mid \lim _{t \rightarrow 0} t \cdot x \in F^{-}\right\}
$$

where $F^{ \pm}$are parts of a partition $W^{\mathbb{C}^{*}}=F^{+} \sqcup F^{-} \sqcup F^{0}$ of the fixed point set $W^{\mathbb{C}^{*}}$ of $W$, satisfying

1. if $u \preceq v$ and $v \in F^{+}, u \in F^{+}$;

2. if $u \preceq v$ and $u \in F^{-}$, then $v \in F^{-}$;

3. if $u \supsetneqq v$ and $v \in F^{0}$, then $u \in F^{+}$;

4. if $u \supsetneqq v$ and $u \in F^{0}$, then $v \in F^{-}$, 
with respect to the ordering $\preceq$ defined as follows: For $x, y \in W^{\mathbb{C}^{*}}, x \preceq y$ if there exists a sequence $x_{1}, \cdots, x_{n} \in W$ such that

$$
\lim _{t \rightarrow 0} t \cdot x_{1}=x, \lim _{t \rightarrow \infty} t \cdot x_{n}=y, \lim _{t \rightarrow \infty} t \cdot x_{k}=\lim _{t \rightarrow 0} t \cdot x_{k+1}, \quad \forall k .
$$

Then $M_{ \pm}=\left[X_{ \pm} / \mathbb{C}^{*}\right]$ is a simple flip. The proof of the simpleness condition is essentially contained in $[22$, Theorem 11.1$]$. Notice that all GIT $\mathbb{C}^{*}$-flips are special cases of this example.

2.3. Simple wall crossings and simple flips. In this subsection, we investigate a case where a simple wall crossing of moduli of derived category objects is a simple flip.

Let $M_{ \pm} \subset \mathcal{M}$ be a moduli of semistable derived category objects as in Definition 2.1. We first show that $\mathcal{M}$ can be written as a global $\mathbb{C}^{*}$ quotient of a separated DM-stack $X$. Fix $B \in K(S)$ that distinguishes elements in $M_{1}$ and $M_{2}$, as stated in Definition 2.1.

Definition 2.6. For $E \in \mathcal{M}$, let

$$
L(E):=\operatorname{det} R \Gamma(E \otimes B)^{\chi(E)} \cdot \operatorname{det} R \Gamma(E)^{-\chi(E \otimes B)},
$$

where $\operatorname{det} R \Gamma(E)=\otimes_{i}\left(\wedge^{t o p} H^{i}(E)\right)^{(-1)^{i}}$.

Observe that if $E=E_{1} \oplus E_{2}$ is strictly $\tau_{0}$-semistable, then any $\varphi \in \operatorname{Aut}(E) / \mathbb{C}^{*}$ $\left(\cong \mathbb{C}^{*}\right)$, where $\mathbb{C}^{*} \leq \operatorname{Aut}(E)$ is the subgroup $\mathbb{C}^{*} \cdot \operatorname{id}_{E}$, has a representative $\lambda \cdot \operatorname{id}_{E_{1}} \oplus$ $\lambda^{-1} \cdot \operatorname{id}_{E_{2}}$. The weight of this circle action on $L(E)$ is

$$
\nu:=\left(\chi\left(E_{1} \otimes B\right)-\chi\left(E_{2} \otimes B\right)\right) \cdot \chi(E) \neq 0 .
$$

Note that by assumption, $\chi(E)=2 \chi\left(E_{1}\right)=2 \chi\left(E_{2}\right) \neq 0$.

Note that the order $M_{1}$ and $M_{2}$ is determined by the choice of $\tau_{+}$and $\tau_{-}$according to Definition 2.1. Thus by interchanging $\tau_{+}$and $\tau_{-}$if necessary, we may and will assume $\nu>0$.

Definition 2.7. Two pairs $\left(E_{1}, \sigma_{1}\right)$ and $\left(E_{2}, \sigma_{2}\right)$ where $\sigma_{i}: L\left(E_{i}\right) \stackrel{\cong}{\longrightarrow}$, are isomorphic if there exists a $\varphi: E_{1} \stackrel{\stackrel{ }{\longrightarrow}}{\longrightarrow} E_{2}$ such that $\sigma_{2} \circ L(\varphi)=\sigma_{1}$.

The automorphism group $\operatorname{Aut}(E, \sigma)$ of such a pair $(E, \sigma)$ is the group of all isomorphisms from $(E, \sigma)$ to itself. Obviously, $\mathbb{C}^{*} \cdot \operatorname{id}_{E} \leq \operatorname{Aut}(E, \sigma)$.

Lemma 2.8. $\operatorname{Aut}(E, \sigma) / \mathbb{C}^{*} \cdot \operatorname{id}_{E}$ is a finite group.

Proof. Unless $E=E_{1} \oplus E_{2}$, the quotient group is trivial. When $E=E_{1} \oplus E_{2}$, any $\lambda \cdot \operatorname{id}_{E_{1}} \oplus \lambda^{-1} \cdot \operatorname{id}_{E_{2}} \in \operatorname{Aut}(E, \sigma) / \mathbb{C}^{*}$ should satisfy $\lambda^{\nu}=1$ with $\nu$ defined in (2.3); hence the group is finite.

Proposition 2.9. Let $M_{ \pm} \subset \mathcal{M}$ be as in Definition 2.1 and suppose for $E \in \mathcal{M}$ $\operatorname{Ext}^{\leq-1}(E, E)=0$.

(1) The groupoid $X$ of families of pairs $(E, \sigma)$ of $E \in \mathcal{M}$ and $\sigma: L(E) \cong \mathbb{C}$ is a separated DM-stack with a strict representable $\mathbb{C}^{*}$-action by $t \cdot(E, \sigma)=$ $(E, t \sigma)$; the fixed point locus is $M_{1} \times M_{2}=\left\{\left(E_{1} \oplus E_{2}, \sigma\right) \mid E_{i} \in M_{i}\right\} / \cong$.

\footnotetext{
${ }^{1}$ The strictness is defined in $[20] ;$ the $\mathbb{C}^{*}$-action is representable if the multiplication morphism $\mathbb{C}^{*} \times X \rightarrow X$ is representable.
} 
(2) $\mathcal{M} \cong\left[X / \mathbb{C}^{*}\right]$; let $X_{ \pm} \subset X$ be as defined in $(2.2)$, then $M_{ \pm} \cong\left[X_{ \pm} / \mathbb{C}^{*}\right]$;

(3) The pair $M_{ \pm} \cong\left[X_{ \pm} / \mathbb{C}^{*}\right]$ is a simple flip (Definition 2.2).

Proof. By [14], $\mathcal{M}$ is an Artin stack locally of finite presentation. The relative version of the construction of $L(E)$ from $E$ shows that $X$ is an Artin stack. Since every $(E, \sigma) \in X$ has finite stabilizer, $X$ is actually a DM-stack by slice argument. The strictness and the representable property of the $\mathbb{C}^{*}$-action follows directly from the construction.

It follows from the property of stability and from Definition 2.1 that $X^{\mathbb{C}^{*}}$ consist of $E_{1} \oplus E_{2}, E_{i} \in M_{i}$. We now prove that $(E, \sigma) \in X_{ \pm}$if and only if $E$ is $\tau_{ \pm}$-stable. For this, we need a description of $\Sigma_{ \pm}^{\circ}$.

Let $E \in M_{+}$be $\tau_{+}$-stable but not $\tau_{0}$-stable. By Definition 2.1, $E$ is a non-trivial extension of an $E_{2} \in M_{2}$ by an $E_{1} \in M_{1}$. Let $\alpha \in \operatorname{Ext}^{1}\left(E_{2}, E_{1}\right)$ be the extension class of the distinguished triangle $E_{1} \rightarrow E \rightarrow E_{2} \rightarrow$. Let $0 \in \mathbb{A}^{1}$ be the origin; let $e \in \Gamma\left(\mathcal{O}_{\mathbb{A}^{1}}(0)\right)$ be the image of $1 \in \Gamma\left(\mathcal{O}_{\mathbb{A}^{1}}\right) \subset \Gamma\left(\mathcal{O}_{\mathbb{A}^{1}}(0)\right)$. Then $e$ vanishes simply at $0 \in \mathbb{A}^{1}$. Let $p_{S}$ and $p_{\mathbb{A}^{1}}$ be the projections of $S \times \mathbb{A}^{1}$. Then

$$
\alpha \cdot e \in \operatorname{Ext}_{S \times \mathbb{A}^{1}}^{1}\left(p_{S}^{*} E_{2} \otimes p_{\mathbb{A}^{1}}^{*} \mathcal{O}_{\mathbb{A}^{1}}(-0), p_{S}^{*} E_{1}\right)=\operatorname{Ext}^{1}\left(E_{2}, E_{1}\right) \otimes \Gamma\left(\mathcal{O}_{\mathbb{A}^{1}}(0)\right)
$$

defines an $\mathcal{E} \in D^{b}\left(\operatorname{Coh}\left(S \times \mathbb{A}^{1}\right)\right)$ that fits into the following diagram of distinguished triangles

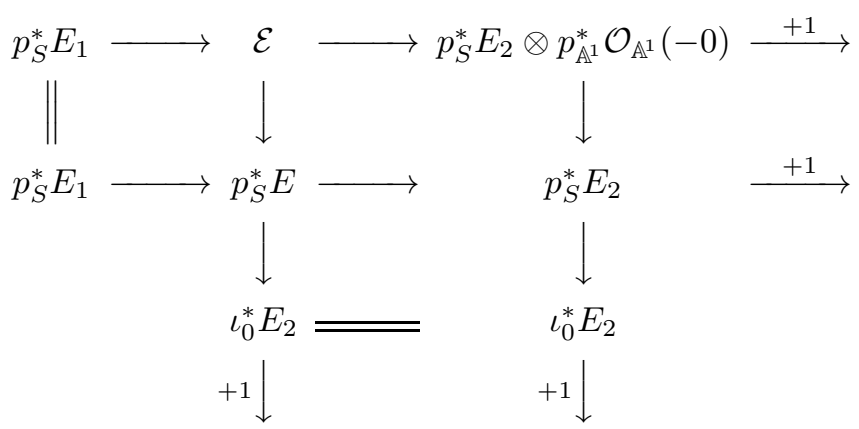

where $\iota_{0}: S \times 0 \longrightarrow S \times \mathbb{A}^{1}$ is the central fiber.

We now form a line bundle on $\mathbb{A}^{1}$

$$
L(\mathcal{E})=\operatorname{det} R p_{\mathbb{A}^{1} *}\left(\mathcal{E} \otimes p_{S}^{*} B\right)^{\chi(E)} \otimes \operatorname{det} R p_{\mathbb{A}^{1} *}(\mathcal{E})^{-\chi(E \otimes B)} .
$$

Using (2.4), we obtain canonical isomorphisms

$$
L(\mathcal{E}) \otimes L\left(\iota_{0}^{*} E_{2}\right) \cong L\left(p_{S}^{*} E\right) \cong L(E) \otimes \mathcal{O}_{\mathbb{A}^{1}} .
$$

Since canonically $L\left(\iota_{0}^{*} E_{2}\right) \cong \mathcal{O}_{\mathbb{A}^{1}}\left(-\nu^{\prime} 0\right)$, where $\nu^{\prime}=\frac{1}{2} \nu$ and $\nu>0$ is as in (2.3), we have

$$
L(\mathcal{E}) \cong L(E) \otimes \mathcal{O}_{\mathbb{A}^{1}}\left(\nu^{\prime} 0\right) .
$$

Now let $\sigma: L(E) \rightarrow \mathbb{C}$ be a fixed isomorphism; we fix an isomorphism $\tilde{\sigma}: L(\mathcal{E}) \cong$ $\mathcal{O}_{\mathbb{A}^{1}}$. Then restricting the isomorphism (2.5) to $\mathbb{A}^{1}-0$, for a $u \in \Gamma\left(\mathcal{O}_{\mathbb{A}^{1}}\right)$ that vanishes simply at $0 \in \mathbb{A}^{1}$ we have

$$
\tilde{\sigma}=u^{\nu^{\prime}} \cdot \sigma
$$


Since $\left.\left.\mathcal{E}\right|_{S \times\left(\mathbb{A}^{1}-0\right)} \cong p_{S}^{*} E\right|_{S \times\left(\mathbb{A}^{1}-0\right)}$ and $\iota_{0}^{*} \mathcal{E}=E_{1} \oplus E_{2}$, this proves $\lim _{t \rightarrow 0} t \cdot(E, \sigma)=$ $\left(E_{1} \oplus E_{2},\left.\tilde{\sigma}\right|_{0}\right) \in X^{\mathbb{C}^{*}}$. Hence $\tau_{+}$-stable but not $\tau_{0}$-stable objects are all over $\Sigma_{+}^{\circ}$.

For the other direction, suppose $(E, \sigma) \in X-X^{\mathbb{C}^{*}}$ such that

$$
\lim _{t \rightarrow 0} t \cdot(E, \sigma)=\left(E_{1} \oplus E_{2}, \cdot\right) \in X^{\mathbb{C}^{*}} .
$$

Let $R=\mathbb{C}[t]_{(t)}$, localized at the ideal $(t)$. We let $(\mathcal{E}, \tilde{\sigma})$ on $S \times \operatorname{Spec} R$ be given by the morphism Spec $R \rightarrow X$ that is given by the completion of the morphism $\mathbb{C}^{*}=\left\{t \cdot(E, \sigma) \mid t \in \mathbb{C}^{*}\right\} \rightarrow X$. Here the completion exists because of (2.6). As usual, we denote by $\xi$ and $\xi_{0}$ the generic and the closed points of Spec $R$. By the construction of $\mathcal{E}$, we have isomorphism

$$
\varphi_{\xi}:\left.\left.\mathcal{E}\right|_{S \times \xi} \stackrel{\cong}{\longrightarrow} p_{S}^{*} E\right|_{S \times \xi} \quad \text { where } p_{S}: S \times \operatorname{Spec} R \rightarrow S .
$$

Thus $\operatorname{Hom}_{S \times \operatorname{Spec} R}\left(p_{S}^{*} E, \mathcal{E}\right)$ is a non-trivial $R$-module. Let $\varphi \in \operatorname{Hom}_{S \times \operatorname{Spec} R}\left(p_{S}^{*} E, \mathcal{E}\right)$ be such that $\left.\varphi\right|_{S \times \xi}=\varphi_{\xi}$. By replacing $\varphi$ by $t^{c} \varphi$ for some $c \leq 0$, we can assume that $\left.\varphi\right|_{S \times \xi_{0}}$ is non-trivial. Namely, for the closed embedding $\iota: S \times \xi_{0} \rightarrow S \times \operatorname{Spec} R$, $\iota^{*} \varphi: \iota^{*} \mathcal{E}=E_{1} \oplus E_{2} \rightarrow E$ is non-trivial.

Since $(E, \sigma) \notin X^{\mathbb{C}^{*}}$, since there is a non-trivial $E_{1} \oplus E_{2} \rightarrow E$ and since both $E_{i}$ are $\tau_{0}$-stable and $E$ is $\tau_{0}$-semistable, $E$ is either an extension of $E_{2}$ by $E_{1}$ or vice versa. By a parallel discussion as we did above, $E$ must be an extension of $E_{2}$ by $E_{1}$. This proves that $X_{-}=X-\Sigma_{+}$. The same holds for $X_{+}$. This proves (2).

Finally, it is direct to check that (3) holds for $X$, knowing that $M_{ \pm}$are proper and separated.

We prove the simpleness condition in the next Lemma.

Lemma 2.10. The pair $X_{ \pm} \subset X$ satisfies the Simpleness Condition.

Proof. We let $f_{ \pm}$and $g$ be as in the statement of Simpleness Condition 2.3. We let $z_{0}=\lim _{t \rightarrow 0} t \cdot f_{+}\left(\xi_{0}\right), z_{0} \in X^{\mathbb{C}^{*}}$ by our construction of $\Sigma_{+}^{\circ}$. We pick an affine étale $\rho: U \rightarrow X$ that covers $z_{0}$. We let $\bar{U}$ be a compactification of $U$.

We next let $p_{1}$ and $p_{2}$ be the first and the second projections of $\operatorname{Spec} R \times \mathbb{C}^{*}$. We form the morphism

$$
F^{\prime}=p_{2} \cdot\left(f_{+} \circ p_{1}\right): \operatorname{Spec} R \times \mathbb{C}^{*} \rightarrow X,
$$

where - denotes the group action. Since the closure of the image of $F^{\prime}$ in $X$ contains $z_{0}$, there is an open $V^{\prime} \subset \operatorname{Spec} R \times \mathbb{C}^{*}$ such that $\left(\xi_{0}, c\right) \in V^{\prime}$ for general closed $c \in \mathbb{C}^{*}$ and $F^{\prime}\left(V^{\prime}\right)$ is contained in the image $\rho(U) \subset X$.

Thus by replacing $R$ by a finite extension of $R$, which we still denote by $R$, we can assume that there is an open $V \subset \operatorname{Spec} R \times \mathbb{C}^{*}$ so that $\left(\xi_{0}, c\right) \in V$ for general closed $c \in \mathbb{C}^{*} ; F^{\prime}$ lifts to an $F: V \rightarrow U$ so that we have a commutative diagram

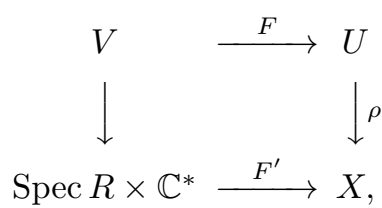

and $\lim _{t \rightarrow 0} F\left(\left(\xi_{0}, t\right)\right)=\overline{z_{0}} \in U$ with $\rho\left(\overline{z_{0}}\right)=z_{0}$.

We remark that with the new $R$, the induced morphisms $f_{ \pm}$: Spec $R \rightarrow X$ satisfy the same property, except that the integer $a$ is replaced by its multiple by the 
ramification index of the finite extension. Thus the simpleness condition is invariant under finite extensions of $R$.

We then let $S=\operatorname{Spec} R \times \mathbb{P}^{1}$, which contains $\operatorname{Spec} R \times \mathbb{C}^{*}$ via $\mathbb{C}^{*}=\mathbb{A}^{1}-\{0\} \subset$ $\mathbb{A}^{1} \subset \mathbb{P}^{1}$. Since $S$ is a smooth surface, there is a minimal finite set $A_{0} \subset S$ so that $F$ extends to

$$
F_{0}: S \backslash A_{0} \longrightarrow \bar{U} .
$$

(We call $A_{0}$ the indeterminacy locus of $F_{0}$.) We let $\mathbb{C}^{*}$ act on $S$ by acting trivially on $\operatorname{Spec} R$ and tautologically on $\mathbb{C}^{*}$ (with weight 1 ).

We now partially resolve the indeterminacy of $F_{0}$. We let $A_{0}^{\prime}=A_{0} \cap S^{\mathbb{C}^{*}}$. We blow up $S$ along $A_{0}^{\prime}$, obtaining $S_{1}$, which has an induced $\mathbb{C}^{*}$-action. We let $A_{1} \subset S_{1}$ be the indeterminacy locus of the extension $F_{1}: S_{1} \backslash A_{1} \rightarrow \bar{U}$ of $F_{0}$. We then let $A_{1}^{\prime}=A_{1} \cap S_{1}^{\mathbb{C}^{*}}$, blow up $S_{1}$ along $A_{1}^{\prime}$ to obtain $S_{2}$, etc. We continue this process until we get $S_{k} \rightarrow S_{k-1}$ so that the indeterminacy $A_{k}$ of the extension

$$
F_{k}: S_{k} \backslash A_{k} \rightarrow \bar{U}
$$

has no point fixed by $\mathbb{C}^{*}$.

We let $c \in \mathbb{C}^{*}$ be a general closed point and consider the morphism $\varphi_{1}: \operatorname{Spec} R \rightarrow$ $S$ defined via $\varphi_{1}(\xi)=(\xi, c)$. We let $\eta_{1}:=\varphi_{1}\left(\xi_{0}\right)=\left(\xi_{0}, c\right) \in S$. Since $\left(\xi_{0}, c\right) \in V$ by our choice of $V$, we can assume $\eta_{1} \in S \backslash A_{0}$. Also since $f_{+}\left(\xi_{0}\right) \in \Sigma_{+}^{\circ}$, which is not fixed by $\mathbb{C}^{*}, \eta_{1} \in S$ is not fixed by $\mathbb{C}^{*}$. Since $S_{k}$ is the successive blow-up of $A_{i}^{\mathbb{C}^{*}}$, we can view $\eta_{1}$ as an element in $S_{k}$. Thus $\eta_{1} \in S_{k}-S_{k}^{\mathbb{C}^{*}} \cup A_{k}$.

We next let $\iota:$ Spec $K \rightarrow \operatorname{Spec} R$ be the inclusion, and form $(\iota, g): \operatorname{Spec} K \rightarrow$ Spec $R \times \mathbb{C}^{*}$, where $g$ is given in the statement of the Lemma. We let $\varphi_{-}:$Spec $R \rightarrow S_{k}$ be the extension of $(\iota, g)$. Since $\tilde{p}_{1}: S_{k} \rightarrow$ Spec $R$ induced by the first projection $p_{1}: S \rightarrow \operatorname{Spec} R$ is proper, $\varphi_{-}$exists. We let $\eta_{-}=\varphi_{-}\left(\xi_{0}\right) \in S_{k}$.

We now find a chain of $\mathbb{C}^{*}$-invariant rational curves that connects $\eta_{1}$ and $\eta_{-}$in $S_{k}$. Let $\pi: S_{k} \rightarrow S$ be the blow-up morphism. Let $D=\pi^{-1}\left(\xi_{0} \times \mathbb{P}^{1}\right) ; D$ is connected and is a union of $\mathbb{C}^{*}$-invariant rational curves. As we have argued, $\eta_{1}$ does not lie in the exceptional divisor of $\pi$, thus $\eta_{1}$ lies in a unique $B_{1} \cong \mathbb{P}^{1} \subset D$. Since $D$ is connected and since $\eta_{-} \in D$, we can find a chain of rational curves $B_{1}, \cdots, B_{l}$ so that $q_{i}=B_{i} \cap B_{i+1} \neq \emptyset$ and $\eta_{-} \in B_{l}$.

It is easy to describe the group action on $B_{i}$. Let $\eta_{i}, i \geq 2$, be a closed point in $B_{i}-B_{i}^{\mathbb{C}^{*}}$. Since $\eta_{1}$ is not fixed by $\mathbb{C}^{*}$, we can find $\epsilon \in\{1,-1\}$ so that $\lim _{t \rightarrow 0} t^{\epsilon} \cdot \eta_{1}=q_{1}$. Since the $\mathbb{C}^{*}$-action on Spec $R \times \mathbb{P}^{1}$ is via weight 0 and 1 on the two factors, since $S_{k}$ is derived by successively blowing up a collection of $\mathbb{C}^{*}$-fixed points, and since $B_{i}$ is a chain of $\mathbb{C}^{*}$-invariant rational curves, we must have $\lim _{t \rightarrow 0} t^{\epsilon} \cdot \eta_{i}=q_{i}$ for $i \leq l$. (We let $q_{l}$ be such that $B_{l}^{\mathbb{C}^{*}}=\left\{q_{l-1}, q_{l}\right\}$.) Because $g(\xi) \cdot f_{+}(\xi)=f_{-}(\xi)$ with $g^{*}(t)=\alpha \cdot \zeta^{a}$ and $a>0$, we have $\epsilon>0$. This proves $\epsilon=1$.

We claim that $\left.F_{k}\right|_{B_{1}} \neq$ const. Because $f_{+}\left(\xi_{0}\right) \in \Sigma_{-}^{\circ}$, and because $\rho\left(F_{k}\left(\eta_{1}\right)\right)=$ $f_{+}\left(\xi_{0}\right), \rho\left(F_{k}\left(\eta_{1}\right)\right) \in \Sigma_{+}^{\circ}$. Since $\left.F_{k}\right|_{B_{1}}$ is $\mathbb{C}^{*}$-equivariant, $\left.F_{k}\right|_{B_{1}} \neq$ const. This proves the claim.

We let $\tilde{V}=F_{k}^{-1}(U) \subset S_{k}$. By (2.8), $q_{1} \in V \subset \tilde{V}$. In general, if for some $2 \leq i \leq l$ we know $q_{i-1} \in \tilde{V}$, then $B_{i} \cap \tilde{V} \neq \emptyset$; then we can talk about whether $\left.F_{k}\right|_{B_{i}}$ is constant; if in addition $\left.F_{k}\right|_{B_{i}}$ is constant, then $q_{i} \in \tilde{V}$. Thus by an induction argument, we see that there is an $2 \leq m \leq l+1$ so that (1) $\left.F_{k}\right|_{B_{i}}$ is constant for all $2 \leq i<m$, and $\left.F_{k}\right|_{B_{m}}$ is not constant in case $m \neq l+1$; and (2) $B_{i} \subset \tilde{V}$ for $2 \leq i<m$ and $B_{m} \cap \tilde{V} \neq \emptyset$. 
We now prove item (a) of the simpleness condition. Let $m$ be the integer specified. We claim that in case (a), $m \leq l$. Suppose not, then $F_{k}\left(B_{i}\right)=F_{k}\left(q_{1}\right) \subset U$ for all $2 \leq i \leq l$. Since $\rho$ is étale, this implies $\rho\left(F_{k}\left(B_{i}\right)\right) \subset X^{\mathbb{C}^{*}}$ for the same $i$; hence $\rho\left(\bar{F}_{k}\left(\eta_{-}\right)\right) \in X^{\mathbb{C}^{*}}$. But this contradicts $\rho\left(F_{k}\left(\eta_{-}\right)\right)=f_{-}\left(\xi_{0}\right)$ and the assumption $f_{-}\left(\xi_{0}\right) \in \Sigma_{-}^{\circ}$. Therefore, $m \leq l$ and consequently, $\left.F_{k}\right|_{B_{m}}$ is not constant. Since $B_{m} \cap \tilde{V} \neq \emptyset$, we may let $\eta_{m} \in B_{m} \cap \tilde{V}$ be a general closed point.

We claim that $\rho\left(F_{k}\left(\eta_{m}\right)\right) \in \Sigma_{-}^{\circ}$. By the definition of $\Sigma_{-}^{\circ}, \rho\left(F_{k}\left(\eta_{m}\right)\right) \in \Sigma_{-}^{\circ}$ if $\rho\left(F_{k}\left(\eta_{m}\right)\right) \notin X^{\mathbb{C}^{*}}$ and $\lim _{t \rightarrow 0} t^{-1} \cdot \rho\left(F_{k}\left(\eta_{m}\right)\right) \in X^{\mathbb{C}^{*}}$. Suppose $\rho\left(F_{k}\left(\eta_{m}\right)\right) \in X^{\mathbb{C}^{*}}$. As argued before, $\rho \circ F_{k}$ is $\mathbb{C}^{*}$-equivariant (where it is defined), and $\rho$ étale. Thus $\rho\left(F_{k}\left(\eta_{m}\right)\right) \in X^{\mathbb{C}^{*}}$ implies that $\left.F_{k}\right|_{B_{m}}$ is constant, a contradiction. For the remaining condition, we notice that

$$
\lim _{t \rightarrow 0} t^{-1} \cdot \rho\left(F_{k}\left(\eta_{m}\right)\right)=\lim _{t \rightarrow 0} \rho\left(F_{k}\left(t^{-1} \cdot \eta_{m}\right)\right)=\rho\left(F_{k}\left(q_{m-1}\right)\right)=z_{0} \in X^{\mathbb{C}^{*}} .
$$

This proves that $\rho\left(F_{k}\left(\eta_{m}\right)\right) \in \Sigma_{-}^{\circ}$.

Then since both $\left[f_{-}\left(\xi_{0}\right)\right]$ and $\left[\rho\left(F_{k}\left(\eta_{m}\right)\right)\right] \in\left[X_{-} / \mathbb{C}^{*}\right]$ are specializations of $\left[f_{-}(\xi)\right]=\left[f_{+}(\xi)\right] \in\left[X_{-} / \mathbb{C}^{*}\right]$, by the assumption that $\left[X_{-} / \mathbb{C}^{*}\right]$ is separated, we conclude that

$$
\left[f_{-}\left(\xi_{0}\right)\right]=\left[\rho\left(F_{k}\left(\eta_{m}\right)\right)\right] \in\left[X_{-} / \mathbb{C}^{*}\right]
$$

We prove $l=m$. For this, we use the properties of the construction of $X$. Suppose $l>m$. Since $S_{k}$ is the result of successive blow-ups of $\mathbb{C}^{*}$-fixed points of $S=$ Spec $R \times \mathbb{P}^{1}$, for the $\eta_{m} \in B_{m}$, there are a finite extension $\tilde{R} \supset R$ and a morphism $\tilde{e}: \operatorname{Spec} \tilde{R} \rightarrow \mathbb{C}^{*}$ so that $\tilde{e} \cdot \tilde{\varphi}_{-}$extends to $\tilde{\varphi}_{m}: \operatorname{Spec} \tilde{R} \rightarrow S_{k}$ so that $\tilde{\varphi}_{m}\left(\tilde{\xi}_{0}\right)=\eta_{m}$. By the property of the $\mathbb{C}^{*}$-actions on the chain $B_{i}$, we have $\tilde{e}^{*}(t)=\tilde{\beta} \cdot \tilde{\zeta}^{r}$ with $r<0$ and $\tilde{\beta} \in \tilde{R}$ invertible.

Since $\eta_{m} \in \tilde{V}, \rho \circ F_{k} \circ \tilde{\varphi}_{m}: \operatorname{Spec} \tilde{R} \rightarrow X$ is defined. Let

$$
\tilde{f}_{m}:=\rho \circ F_{k} \circ \tilde{\varphi}_{m}: \operatorname{Spec} \tilde{R} \longrightarrow X \text {. }
$$

(Let $\tilde{f}_{-}$be the composite of $\operatorname{Spec} \tilde{R} \rightarrow \operatorname{Spec} R$ with $f_{-}$; cf. comments after Definition 2.2.) Then $\tilde{e} \cdot \tilde{f}_{-}=\tilde{f}_{m}$.

Let $\left(E_{m}, \sigma_{m}\right)$ and $\left(E_{-}, \sigma_{-}\right)$be families on $S \times \operatorname{Spec} \tilde{R}$ that are the pull-backs of the universal family of $X$ via $\tilde{f}_{m}$ and $\tilde{f}_{-}$, respectively. Let $\iota: S \times \tilde{\xi}_{0} \rightarrow S \times \operatorname{Spec} \tilde{R}$ be the closed embedding. Because $\tilde{e} \cdot \tilde{f}_{-}=\tilde{f}_{m}$, the families $E_{m}$ and $E_{-}$restricted to $S \times \tilde{\xi}$ are isomorphic; let $\alpha:\left.\left.E_{-}\right|_{S \times \tilde{\xi}} \rightarrow E_{m}\right|_{S \times \tilde{\xi}}$ be such an isomorphism. By the property of the line bundle $L(\cdot), \alpha$ induces an isomomorphism $L(\alpha):\left.\left.L\left(E_{-}\right)\right|_{\tilde{\xi}} \rightarrow L\left(E_{m}\right)\right|_{\tilde{\xi}}$.

Recall that $\sigma_{m}: L\left(E_{m}\right) \rightarrow \mathcal{O}_{\text {Spec } \tilde{R}}$ and $\sigma_{-}: L\left(E_{-}\right) \rightarrow \mathcal{O}_{\text {Spec } \tilde{R}}$ are isomorphisms. By the construction of the $\mathbb{C}^{*}$-action on $X, \tilde{e} \cdot \tilde{f}_{-}=\tilde{f}_{m}$ implies that $\left.\tilde{e} \cdot \sigma_{-}\right|_{\tilde{\xi}}=\left.\sigma_{m}\right|_{\tilde{\xi}}$. Therefore,

$$
\left.\sigma_{m}\right|_{\tilde{\xi}} \circ L(\alpha) \circ\left(\left.\sigma_{-}\right|_{\tilde{\xi}}\right)^{-1}=\tilde{e}: \mathcal{O}_{\operatorname{Spec} \tilde{K}} \longrightarrow \mathcal{O}_{\operatorname{Spec} \tilde{K}},
$$

which is $\tilde{\beta} \cdot \tilde{\zeta}^{r}$ with $r<0$. On the other hand, by scaling $\alpha$ by a power of $\tilde{\zeta}$, say $\tilde{\zeta}^{d}$, we can assume that $\tilde{\zeta}^{d} \cdot \alpha$ extends to an $\tilde{\alpha}: E_{-} \rightarrow E_{m}$ so that $\iota^{*} \tilde{\alpha}: \iota^{*} E_{-} \rightarrow \iota^{*} E_{m}$ is nontrivial. By $(2.9), \iota^{*} E_{m} \cong \iota^{*} E_{-}$and they are $\tau_{-}$-stable. Thus $\iota^{*} \tilde{\alpha}$ is an isomorphism. Therefore, $\tilde{\alpha}$ is an isomorphism and

$$
\sigma_{m} \circ L(\tilde{\alpha}) \circ \sigma_{-}^{-1}: \mathcal{O}_{\mathrm{Spec} \tilde{R}} \longrightarrow \mathcal{O}_{\mathrm{Spec} \tilde{R}}
$$


is an isomorphism.

Finally, since $L(\alpha)$ is independent of scaling,

$$
\left.\sigma_{m}\right|_{\tilde{\xi}} \circ L(\alpha) \circ\left(\left.\sigma_{-}\right|_{\tilde{\xi}}\right)^{-1}=\left.\sigma_{m}\right|_{\tilde{\xi}} \circ L\left(t^{d} \cdot \alpha\right) \circ\left(\left.\sigma_{-}\right|_{\tilde{\xi}}\right)^{-1}=\left.\sigma_{m}\right|_{\tilde{\xi}} \circ L(\tilde{\alpha}) \circ\left(\left.\sigma_{-}\right|_{\tilde{\xi}}\right)^{-1} .
$$

Since the right hand side extends to an invertible element in $\tilde{R}$, it contradicts $(2.10)$ and $r<0$. This proves $l=m$.

We now finish the proof of (a). Let $\tilde{h}: \operatorname{Spec} \tilde{K} \rightarrow \mathbb{C}^{*}$ be given by $\tilde{h}^{*}(t)=\beta \cdot t^{b}$ with $0<b<\imath(\tilde{R}) \cdot a$. We let $\tilde{\varphi}_{b}: \operatorname{Spec} \tilde{R} \rightarrow S_{k}$ be the extension of $\tilde{h} \cdot \tilde{\varphi}_{1}: \operatorname{Spec} K \rightarrow S_{k}$. Since $\tilde{g} \cdot \tilde{\varphi}_{1}$ extends to $\tilde{\varphi}_{-}$with $\tilde{\varphi}_{-}\left(\tilde{\xi}_{0}\right) \in B_{l}, 0<b<\imath(\tilde{R}) \cdot a$ guarantees that $\tilde{\varphi}_{b}$ exists and $\tilde{\varphi}_{b}\left(\tilde{\eta}_{0}\right) \in B_{2} \cup \cdots \cup B_{m-1}$. Since for $2 \leq i \leq m-1, B_{i} \subset \tilde{V}$ and $F_{k}\left(B_{i}\right)=F_{k}\left(q_{1}\right), \rho \circ F_{k} \circ \tilde{\varphi}_{b}$ is defined and $\rho \circ F_{k} \circ \tilde{\varphi}_{b}\left(B_{i}\right)=z_{0} \in X^{\mathbb{C}^{*}}$. Since $\tilde{h} \cdot \tilde{f}_{+}=\rho \circ F_{k} \circ\left(\tilde{h} \cdot \tilde{\varphi}_{1}\right): \operatorname{Spec} \tilde{K} \rightarrow X, \tilde{h} \cdot \tilde{f}_{+}$extends to $\left(\tilde{h} \cdot \tilde{f}_{+}\right)^{\#}:=\rho \circ F_{k} \circ \tilde{\varphi}_{b}$ such that $\left(\tilde{h} \cdot \tilde{f}_{+}\right)^{\#}\left(\tilde{\xi}_{0}\right)=z_{0} \in X^{\mathbb{C}^{*}}$. This proves $(\mathrm{a})$.

The proof of (b) is similar and we omit it.

2.4. Symmetric obstruction theory. In this subsection, we discuss symmetric obstruction theory for simple flips. Let $X$ be a DM-stack acted on by $\mathbb{C}^{*}$. Let $\mathcal{M}=\left[X / \mathbb{C}^{*}\right]$ be the quotient stack.

Definition 2.11. ([8]) We say the quotient stack $\mathcal{M}=\left[X / \mathbb{C}^{*}\right]$ has a perfect obstruction theory (resp. symmetric obstruction theory) if $X$ has a $\mathbb{C}^{*}$-equivariant perfect (resp. symmetric) obstruction theory.

We recall that as part of the definition, there is a $\mathbb{C}^{*}$-equivariant derived category object $F^{\bullet} \in D_{\mathbb{C}^{*}}^{b}(\operatorname{Coh} X)$, which étale locally is quasi-isomorphic to two term complexes of locally free sheaves, and an arrow $F^{\bullet} \rightarrow \mathbb{L}_{\bar{X}}^{\geq-1}$ satisfying the requirement of perfect obstruction theory or symmetric obstruction theory. (Here $\mathbb{L}_{X}^{\geq-1}$ is the truncation of the cotangent complex of $X$.)

REMARK 2.12. For moduli of stable sheaves, analytic locally we may find a function $f$ such that locally the moduli space and its obstruction theory is given by the vanishing of the differential $d f$ (cf. [10]). We call such functions $f$ local ChernSimons functionals of the moduli space. For more general moduli problems like stable objects in the derived category, local Chern-Simons functionals are not known to exist. See [15, Appendix A] for a discussion about the existence of Chern-Simons functional.

Lemma 2.13. Let $S$ be a Calabi-Yau three-fold. Let $M_{ \pm}=\left[X_{ \pm} / \mathbb{C}^{*}\right]$ be a simple wall crossing in $\mathcal{M}=\left[X / \mathbb{C}^{*}\right]$ of moduli of stable objects in $D^{b}(\mathrm{Coh} S)$ (Definition 2.1) such that $E x t^{\leq-1}(E, E)=0$ for any $E \in \mathcal{M}$. Suppose either $X \mathbb{C}^{*}$-equivariantly embeds in a smooth DM-stack, or $X$ has a global tautological family arising from $X \rightarrow \mathcal{M}$. Then $X$ has a $\mathbb{C}^{*}$-equivariant symmetric obstruction theory.

Proof. Suppose first that we have a tautological object $\mathcal{E} \in D^{b}(\operatorname{Coh}(X \times S))$ induced from $X \rightarrow \mathcal{M}$. Let $\pi: X \times S \rightarrow X$ be the projection. Let $\mathbb{L}_{X}^{\bullet}$ denote the cotangent complex of $X$ and $\mathbb{L}_{\mathcal{M}}$ be defined by the distinguished triangle

$$
\mathbb{L}_{\mathcal{M}} \longrightarrow \mathbb{L}_{X} \longrightarrow \mathcal{O}_{X}
$$

where the last arrow is given by the action of $\mathbb{C}^{*}$ on $X$. Taking the duals, we have a distinguished triangle

$$
\mathcal{O}_{X} \longrightarrow \mathbb{L}_{X}^{\bullet \vee} \longrightarrow \mathbb{L}_{\mathcal{M}}{ }^{\vee} \longrightarrow \mathcal{O}_{X}[1]
$$


By the construction in [9] using the Atiyah class, we have a morphism $\mathbb{L}_{X}{ }^{\vee} \rightarrow$ $R:=R \pi_{*} \mathcal{H} \operatorname{om}(\mathcal{E}, \mathcal{E})_{0}[1]$. Since the $\mathbb{C}^{*}$ action on a pair $(E, \sigma) \in X$ fixes $E$, the composition

$$
\mathcal{O}_{X} \longrightarrow \mathbb{L}_{X}^{\bullet \vee} \longrightarrow R
$$

is trivial and hence we have an induced morphism $\mathbb{L}_{\mathcal{M}}{ }^{\vee} \rightarrow R$. On the other hand, the functorial assignment $E \rightsquigarrow L(E)$ of Definition 2.6 induces a morphism

$$
R=R \pi_{*} \mathcal{H o m}(\mathcal{E}, \mathcal{E})_{0}[1] \longrightarrow \operatorname{Hom}(L(\mathcal{E}), L(\mathcal{E}))[1]=\mathcal{O}_{X}[1] .
$$

Therefore we have a diagram

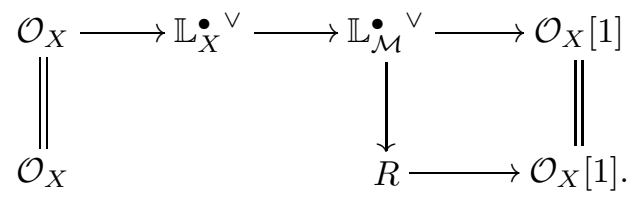

Let $R^{\prime}$ be defined by the distinguished triangle

$$
\mathcal{O}_{X} \longrightarrow R^{\prime} \longrightarrow R \longrightarrow \mathcal{O}_{X}[1]
$$

so that we have a commutative diagram of distinguished triangles

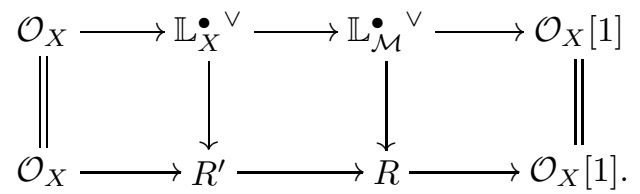

Note that $H^{-1}(R)=\operatorname{Hom}_{\pi}(\mathcal{E}, \mathcal{E})_{0}$ injects into $\mathcal{O}_{X}$ because the stabilizer $\mathbb{C}^{*}$ of an $E_{1} \oplus E_{2} \in M_{1} \times M_{2}$ acts nontrivially on $L\left(E_{1} \oplus E_{2}\right)$. By a simple diagram chase with the long exact sequence, we find that the second vertical morphism $\mathbb{L}_{X}{ }^{\vee} \rightarrow R^{\prime}$ is an obstruction theory of $X$ but it is not perfect since $H^{2}\left(R^{\prime}\right) \cong H^{2}(R)=\operatorname{Ext}_{\pi}^{3}(\mathcal{E}, \mathcal{E})_{0}$ may be non-trivial.

To remove $H^{2}\left(R^{\prime}\right)$, we take the dual $\gamma^{\vee}: \mathcal{O}_{X}[-1] \rightarrow R^{\vee}$ of the bottom right arrow $\gamma: R \rightarrow \mathcal{O}_{X}[1]$ of the above diagram. By the Serre duality $R^{\vee} \cong R[1]$, we obtain

$$
\mathcal{O}_{X}[-2] \stackrel{\gamma^{\vee}[-1]}{\longrightarrow} R^{\vee}[-1] \cong R \stackrel{\nu}{\longrightarrow} \mathcal{O}_{X}[1]
$$

The composition must vanish by a direct check with Serre pairing. From the distinguished triangle $R^{\prime} \rightarrow R \rightarrow \mathcal{O}_{X}[1]$, we therefore obtain a morphism $\mathcal{O}_{X}[-2] \rightarrow R^{\prime}$. Let $R^{\prime \prime}$ be defined by the distinguished triangle

$$
\mathcal{O}_{X}[-2] \longrightarrow R^{\prime} \longrightarrow R^{\prime \prime} \longrightarrow \mathcal{O}_{X}[-1]
$$

Since $H^{-1}(R)=H_{\pi}(\mathcal{E}, \mathcal{E})_{0}$ injects into $\mathcal{O}_{X}, \mathcal{O}_{X}$ surjects onto $H^{2}\left(R^{\prime}\right) \cong H^{2}(R)$. From the long exact sequence, we find that the composition

$$
\mathbb{L}_{X}^{\bullet \vee} \longrightarrow R^{\prime} \longrightarrow R^{\prime \prime}
$$

is a perfect obstruction theory for $X$. By choosing étale locally a free resolution of $R^{\prime \prime}$ and truncating as in the proof of [19, Lemma 2.10], we can find a two-term complex of 
locally free sheaves representing $R^{\prime \prime}$. It is straightforward to see that the Serre duality $R \cong R^{\vee}[-1]$ induces an isomorphism $R^{\prime \prime} \cong R^{\prime \prime} \vee[-1]$ and thus the perfect obstruction theory $\mathbb{L}_{X}{ }^{\vee} \rightarrow R^{\prime \prime}$ is symmetric. This proves the Lemma in case a global tautological family exists.

We next consider the case where $X$ embeds $\mathbb{C}^{*}$-equivariantly into a smooth DMstack, say $X \subset Y$. Let $I$ be the ideal sheaf of $X \subset Y$. Then $\mathbb{L}_{X}^{\geq-1}=\left[I /\left.I^{2} \rightarrow \Omega_{Y}\right|_{X}\right]$. Let $\coprod_{\alpha} Y_{\alpha} \rightarrow Y$ be an affine étale atlas, let $U_{\alpha}=Y_{\alpha} \times_{Y} X \rightarrow X$, so that $U_{\alpha}$ has tautological family $\mathcal{E}_{\alpha}$. The homomorphism $\Omega_{X} \rightarrow \mathcal{O}_{X}$ induced by the group action on $X$ lifts to $\Omega_{U_{\alpha}} \rightarrow \mathcal{O}_{U_{\alpha}}$. Mimicking the prior argument, we see that $U_{\alpha}$ has a symmetric obstruction theory. By [2], the symmetric obstruction theory is given by an almost closed 1-form $\omega_{\alpha} \in \Gamma\left(\Omega_{Y_{\alpha}}\right)$. In particular, $U_{\alpha}=\left(\omega_{\alpha}=0\right)$ and the obstruction is given by

$$
\left[\left.\left.T_{Y_{\alpha}}\right|_{U_{\alpha}} \stackrel{d \omega_{\alpha}}{\longrightarrow} \Omega_{Y_{\alpha}}\right|_{U_{\alpha}}\right] \longrightarrow \mathbb{L}_{\bar{U}_{\alpha}}^{\geq-1}
$$

We remark that if $\omega_{\alpha}^{\prime}$ is another choice of almost closed 1-form giving the symmetric obstruction theory, then necessarily $\omega_{\alpha}-\omega_{\alpha}^{\prime} \in I_{\alpha}^{2}$, where $I_{\alpha}$ is the ideal sheaf of $U_{\alpha} \subset Y_{\alpha}$. Thus $\left.d \omega_{\alpha}\right|_{U_{\alpha}}=\left.d \omega_{\alpha}^{\prime}\right|_{U_{\alpha}}$. Consequently, all $d \omega_{\alpha}$ patch to form a single complex $\left[\left.\left.T_{Y}\right|_{X} \rightarrow \Omega_{Y}\right|_{X}\right]$, and the arrows above patch to form a single arrow

$$
\left[\left.\left.T_{Y}\right|_{X} \longrightarrow \Omega_{Y}\right|_{X}\right] \longrightarrow \mathbb{L}_{X}^{\geq-1} \text {. }
$$

This provides a symmetric obstruction theory of $X$.

3. $\mathbb{C}^{*}$-Intrinsic blow-up. Suppose $X$ has a symmetric obstruction theory, the de facto virtual dimension of $\left[X / \mathbb{C}^{*}\right]$ is -1 . This counters our intuition that both $M_{ \pm} \subset\left[X / \mathbb{C}^{*}\right]$ should have virtual dimension 0 .

As an example, suppose $X \subset Y$ is a $\mathbb{C}^{*}$-equivariant embedding in a smooth scheme and $X=(\omega=0)$, where $\omega \in \Gamma\left(\Omega_{Y}\right)$ is $\mathbb{C}^{*}$-invariant. Then the obstruction theory of $X$ is given by the complex

$$
\left.\mathcal{O}_{X}\left(T_{Y}\right) \stackrel{d \omega}{\longrightarrow} \Omega_{Y}\right|_{X}
$$

Suppose $\mathbb{C}^{*}$ acts on $X$ without fixed points. Then it induces a homomorphism

$$
\eta: \mathcal{O}_{X} \longrightarrow \mathcal{O}_{X}\left(T_{Y}\right)
$$

whose cokernel is the pull-back of the tangent sheaf of $X / \mathbb{C}^{*}$. In principle, we expect that the obstruction complex of $X / \mathbb{C}^{*}$ should be the descent to $X / \mathbb{C}^{*}$ of

$$
\operatorname{coker}\{\eta\} \stackrel{d \omega}{\longrightarrow} \operatorname{ker}\left\{\eta^{\vee}\right\} \text {. }
$$

In this way, the obstruction theory of $X / \mathbb{C}^{*}$ remains symmetric.

This argument breaks down near $X^{\mathbb{C}^{*}}$, where $\eta$ in (3.1) is not a subline bundle. To salvage this argument, we blow up $Y$ along $Y^{\mathbb{C}^{*}}$ and work with a "modified total transform" of $X$ - the modification is to make the resulting scheme independent of the embedding $X \subset Y$. We will call such process the $\mathbb{C}^{*}$-intrinsic blow-up of $X$.

3.1. $\mathbb{C}^{*}$-Intrinsic blow-up. We begin with the easiest case - the formal case.

The formal case: Let $U$ be a formal $\mathbb{C}^{*}$-affine scheme such that its fixed locus $U^{\mathbb{C}^{*}}$ is an affine scheme, and the set of closed points satisfies $\operatorname{Set}(U)=\operatorname{Set}\left(U^{\mathbb{C}^{*}}\right)$. 
Since $U^{\mathbb{C}^{*}}$ is affine, we can embed it into a smooth affine scheme: $U^{\mathbb{C}^{*}} \subset V_{0}$; since $\operatorname{Set}(U)=\operatorname{Set}\left(U^{\mathbb{C}^{*}}\right)$, we can further find a smooth formal $\mathbb{C}^{*}$-scheme $V$ such that the $\mathbb{C}^{*}$-fixed locus $V^{\mathbb{C}^{*}}=V_{0}$, Set $(V)=\operatorname{Set}\left(V_{0}\right)$ and the embedding $U^{\mathbb{C}^{*}} \subset V_{0}$ extends to a $\mathbb{C}^{*}$-equivariant embedding $U \subset V$.

Let $\pi: \bar{V} \rightarrow V$ be the blow-up of $V$ along $V^{\mathbb{C}^{*}}$; let $\tilde{U}=U \times_{V} \bar{V} \subset \bar{V}$ be the total transform. Let $I \subset \mathcal{O}_{V}$ be the ideal sheaf defining $U \subset V$, then $\tilde{U}$ is defined by the ideal sheaf

$$
\tilde{I}:=\pi^{-1}(I) \cdot \mathcal{O}_{\bar{V}}
$$

We let $E \subset \bar{V}$ be the exceptional divisor of $\pi$; let $\xi \in \Gamma\left(\mathcal{O}_{\bar{V}}(E)\right)$ be the defining equation of $E$. Since $U \subset V$ is $\mathbb{C}^{*}$-invariant, $I$ is $\mathbb{C}^{*}$-invariant; thus it admits a weight decomposition $I=I^{\mathbb{C}^{*}} \oplus I^{\mathrm{mv}}$, where $I^{\mathbb{C}^{*}}$ is the $\mathbb{C}^{*}$-invariant part and $I^{\mathrm{mv}}$ consists of nontrivial weight parts of $I$. Clearly, $\pi^{-1}\left(I^{\mathrm{mv}}\right) \subset \xi \cdot \mathcal{O}_{\bar{V}}(-E) \subset \mathcal{O}_{\bar{V}}$; thus $\xi^{-1} \pi^{-1}\left(I^{\mathrm{mv}}\right) \subset \mathcal{O}_{\bar{V}}(-E)$.

We define $\bar{I} \subset \mathcal{O}_{\tilde{V}}$ to be the ideal such that

$$
\bar{I}=\text { the ideal generated by } \pi^{-1}\left(I^{\mathbb{C}^{*}}\right) \text { and } \xi^{-1} \pi^{-1}\left(I^{\mathrm{mv}}\right) \cdot \mathcal{O}_{\bar{V}}(E) \text {. }
$$

We define

$$
\mathrm{bl}_{V}^{\mathbb{C}^{*}} U \subset \bar{V}
$$

to be the subscheme (of $\bar{V}$ ) defined by the ideal $\bar{I}$.

LEMMA 3.1. The scheme $\mathrm{bl}_{V}^{\mathbb{C}^{*}} U$ is independent of the choice of $\mathbb{C}^{*}$-equivariant embedding $U \hookrightarrow V$.

Proof. This is a local problem, thus we only need to prove the case where $U$ is local. (I.e. $U$ contains only one closed point.) We let $\Gamma\left(\mathcal{O}_{V}\right)=A \oplus M$ be the decomposition into the $\mathbb{C}^{*}$ invariant and moving parts; namely $A=\Gamma\left(\mathcal{O}_{V}\right)^{\mathbb{C}^{*}}$ and $M=\Gamma\left(\mathcal{O}_{V}\right)^{\mathrm{mv}}$. We let $\Gamma\left(\mathcal{O}_{U}\right)=B \oplus N$ similarly with $B=\Gamma\left(\mathcal{O}_{U}\right)^{\mathbb{C}^{*}}$ and $N=\Gamma\left(\mathcal{O}_{U}\right)^{\mathrm{mv}}$. Since $V$ is smooth and $A$ is local, without loss of generality we can assume that

$$
\Gamma\left(\mathcal{O}_{V}\right)=A \llbracket y_{\leq n} \rrbracket:=A \llbracket y_{1}, \cdots, y_{n} \rrbracket, \quad y_{1}, \cdots, y_{n} \in \Gamma\left(\mathcal{O}_{V}\right)^{\mathrm{mv}} .
$$

Since $U \rightarrow V$ is a $\mathbb{C}^{*}$-equivariant embedding, we can factor the surjective $\Gamma\left(\mathcal{O}_{V}\right) \rightarrow$ $\Gamma\left(\mathcal{O}_{U}\right)$ as composition

$$
\Gamma\left(\mathcal{O}_{V}\right)=A \llbracket y_{\leq n} \rrbracket \longrightarrow B \llbracket y_{\leq n} \rrbracket \stackrel{\psi_{n}}{\longrightarrow} \Gamma\left(\mathcal{O}_{U}\right)=B \oplus N .
$$

Here $\psi_{n}$ is induced by the restriction $y_{i} \mapsto \psi_{n}\left(y_{i}\right) \in N$.

We next pick a minimal set of generators. Let

$$
m=\operatorname{dim} N /\left(N^{2}, \mathfrak{p} N\right) .
$$

We pick $\alpha_{1}, \cdots, \alpha_{m} \in \Gamma\left(\mathcal{O}_{V}\right)^{\mathrm{mv}}$ such that the homomorphism

$$
\psi_{m}: B \llbracket x_{\leq m} \rrbracket:=B \llbracket x_{1}, \cdots, x_{m} \rrbracket \longrightarrow B \oplus N, \quad x_{i} \mapsto \alpha_{i},
$$

is a surjective ring homomorphism. We let $W=\operatorname{Spec} B \llbracket x_{\leq m} \rrbracket$. Then $\psi_{m}$ defines an embedding $U \subset W$, hence an $\mathbb{C}^{*}$-intrinsic blow-up bl ${ }_{W}^{\mathbb{C}^{*}} U$. 
We pick a homomorphism $\varphi$ (as shown in the diagram)

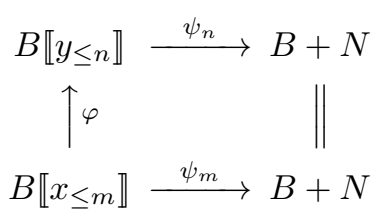

By the the choice of $m(3.3), \varphi$ is injective.

We claim that $\varphi$ induces an isomorphism

$$
\Phi(\varphi): \mathrm{bl}_{V}^{\mathbb{C}^{*}} U \longrightarrow \mathrm{bl}_{W}^{\mathbb{C}^{*}} U
$$

and that it is independent of the choice of $\varphi$.

We first simplify the notation further. We let $a_{i j} \in B$ be so that

$$
\varphi\left(x_{i}\right)=\sum_{j=1}^{n} a_{i j} y_{j} \quad \bmod \left(y_{1}, \cdots, y_{n}\right)^{2}
$$

and that the $m \times m$ matrix $\left(a_{i j}\right)_{1 \leq i, j \leq m}$ is invertible. This is possible by the minimality of $m$ and possibly after re-indexing the $y_{j}$, since $A$ is local. Thus by replacing $y_{j}$ by $\varphi\left(x_{j}\right)$ for $j \leq m$, we can assume $\varphi\left(x_{j}\right)=y_{j}$. Under this arrangement, we see that if we let

$$
\mathcal{I}_{n}:=\psi_{n}^{-1}(0) \subset B \llbracket y_{\leq n} \rrbracket \quad \text { and } \quad \mathcal{I}_{m}:=\psi_{m}^{-1}(0) \subset B \llbracket x_{\leq m} \rrbracket,
$$

then there are $h_{i} \in B \llbracket x_{\leq m} \rrbracket^{\mathrm{mv}}$ such that

$$
\mathcal{I}_{n}=\left(\{\varphi(g)\}_{g \in \mathcal{I}_{m}} \cup\left\{y_{m+i}-\varphi\left(h_{i}\right)\right\}_{1 \leq i \leq n-m}\right) \subset B \llbracket y_{\leq n} \rrbracket .
$$

It is easy to describe the $\mathbb{C}^{*}$-intrinsic blow-ups. First, bl ${ }_{\hat{V}}^{\mathbb{C}^{*}} \hat{U}$ is covered by affines

$$
R_{i}^{n}:=\operatorname{Spec} B\left[v^{i}\right] \llbracket \zeta^{i} \rrbracket /\left(v_{i}^{i}-1\right), \quad v^{i}=\left(v_{1}^{i}, \cdots, v_{n}^{i}\right)
$$

with transitions $R_{i}^{n} \rightarrow R_{j}^{n}$ given by the relation $y_{k}=v_{k}^{i} \zeta^{i}$. The other $\mathrm{bl}_{\hat{W}}^{\mathbb{C}} \hat{U}$ is covered by affines $R_{i}^{m}=\operatorname{Spec} B\left[u^{i}\right] \llbracket \xi^{i} \rrbracket /\left(u_{i}^{i}-1\right)$ similarly defined with $v^{i}$ replaced by $u^{i}=\left(u_{1}^{i}, \cdots, u_{m}^{i}\right)$. To describe the defining equations of $R_{i}^{m} \cap \mathrm{bl}_{\hat{W}}^{\mathbb{C}} \hat{U}$, we introduce the operation of substitution $\mathrm{bl}_{i}$ : for $g=g\left(y_{1}, \cdots, y_{n}\right) \in B \llbracket y_{\leq n} \rrbracket$ with $g(0)=0$, we define

$$
\mathrm{bl}_{i}(g):=\left.\left(\zeta^{i}\right)^{-1} g\left(v_{1}^{i} \zeta^{i}, \cdots, v_{n}^{i} \zeta^{i}\right)\right|_{v_{i}^{i}=1} \in B\left[v^{i}\right] \llbracket \zeta^{i} \rrbracket /\left(v_{i}^{i}-1\right)
$$

The subscheme $R_{i}^{m} \cap \mathrm{bl}_{\hat{W}}^{\mathbb{C}^{*}} \hat{U}$ is then defined by the ideal

$$
\operatorname{bl}_{i}\left(\mathcal{I}_{m}\right)=\left(\left\{\operatorname{bl}_{i}(g): g \in \mathcal{I}_{m}\right\}\right) .
$$

This shows that

$$
R_{i}^{m} \cap \mathrm{bl}_{\hat{W}}^{\mathbb{C}^{*}} \hat{U}=\operatorname{Spec} B\left[u^{i}\right] \llbracket \xi^{i} \rrbracket /\left(u_{i}^{i}-1, \mathrm{bl}_{i}\left(\mathcal{I}_{m}\right)\right)
$$

similarly for $R_{i}^{n} \cap \mathrm{bl}_{\hat{V}}^{\mathbb{C}^{*}} \hat{U}$. 
The homomorphism $\varphi\left(x_{i}\right)=y_{i}$ defines a homomorphism

$$
\Phi_{i}(\varphi): B\left[u^{i}\right] \llbracket \xi^{i} \rrbracket /\left(u_{i}^{i}-1, \mathrm{bl}_{i}\left(\mathcal{I}_{m}\right)\right) \longrightarrow B\left[v^{i}\right] \llbracket \zeta^{i} \rrbracket /\left(v_{i}^{i}-1, \mathrm{bl}_{i}\left(\mathcal{I}_{m}\right)\right)
$$

$\operatorname{via} \xi^{i} \mapsto \zeta^{i}$ and $u_{j}^{i} \mapsto v_{j}^{i}$. Since $y_{m+j}-\varphi\left(h_{j}\right) \in \mathcal{I}_{n}, v_{m+j}^{i}-\operatorname{bl}_{i}\left(\varphi\left(h_{j}\right)\right) \in \operatorname{bl}_{i}\left(\mathcal{I}_{n}\right)$. This proves that $\Phi_{i}(\varphi)$ is surjective. The same argument shows that $\Phi_{i}(\varphi)$ is injective. Thus it is an isomorphism.

We next show that

$$
\mathrm{bl}_{V}^{\mathbb{C}^{*}} U \subset R_{1}^{n} \cup \cdots \cup R_{m}^{n} .
$$

Suppose that there is a closed $b \in \mathrm{bl}_{V}^{\mathbb{C}^{*}} U \cap\left(R_{m+j}^{n}-R_{1}^{n} \cup \cdots \cup R_{m}^{n}\right)$. Let $\left[b_{1}, \cdots, b_{n}\right]$ be the homogeneous coordinates of $b$. Then $b \notin R_{1}^{n} \cup \cdots \cup R_{m}^{n}$ implies that $b_{i}=0$ for $i \leq m$. By the relation $v_{m+j}^{m+i}-\operatorname{bl}_{m+i}\left(\varphi\left(h_{j}\right)\right)$ for $i \geq 1$, we get $b_{m+i}=0$ for all $i \geq 1$. This is a contradiction. This proves the inclusion (3.7).

It is obvious that on restricting to $R_{i}^{n} \cap R_{j}^{n}, \Phi_{i}(\varphi)=\Phi_{j}(\varphi)$. Thus the collection $\left\{\Phi_{i}(\varphi)\right\}_{i \leq m}$ defines an isomorphism

$$
\tilde{\Phi}(\varphi): \mathrm{bl}_{V}^{\mathbb{C}^{*}} U \longrightarrow \mathrm{bl}_{W}^{\mathbb{C}^{*}} U .
$$

Finally, we show that the isomorphism $\tilde{\Phi}(\varphi)$ is independent of the choice of $\varphi$. Notice that this will show that the construction of $\mathrm{bl}_{V}^{\mathbb{C}^{*}} U$ is independent of the choice of the embedding $\psi_{n}$. Suppose $\varphi^{\prime}$ (in place of $\varphi$ in (3.4)) is another homomorphism making the square commutative. We define $\Phi\left(\varphi^{\prime}\right)$, similar to that of $\Phi_{i}(\varphi)$, by sending $x_{j}=u_{j}^{i} \xi^{i}$ to $\left.\varphi^{\prime}\left(x_{j}\right)\right|_{y_{k} \mapsto v_{k}^{i} \zeta^{i}}$. Here the subscript $y_{k} \mapsto v_{k}^{i} \zeta^{i}$ means to substitute the $y_{k}$ variable in the power series $\varphi^{\prime}\left(x_{j}\right)$ by $v_{k}^{i} \zeta^{i}$. It is direct to check that this defines a homomorphism $\Phi_{i}\left(\varphi^{\prime}\right)$, like (3.6).

We claim that for $i \leq m, \Phi_{i}(\varphi)=\Phi_{i}\left(\varphi^{\prime}\right)$. Indeed, by the commutativity of (3.4), there are $g_{i} \in \mathcal{I}_{n}$ such that $\varphi\left(x_{i}\right)-\varphi^{\prime}\left(x_{i}\right)=g_{i}$. Because

$$
\left.\varphi^{\prime}\left(x_{i}\right)\right|_{y_{k} \mapsto v_{k}^{i} \zeta^{i}}=\left.\left(\varphi\left(x_{i}\right)+g_{i}\right)\right|_{y_{k} \mapsto v_{k}^{i} \zeta^{i}} \equiv \zeta^{i} \bmod \zeta^{i} \operatorname{bl}_{i}\left(\mathcal{I}_{n}\right),
$$

we obtain $\Phi_{i}\left(\varphi^{\prime}\right)\left(\xi^{i}\right)=\Phi_{i}(\varphi)\left(\xi^{i}\right)$. For the same reason, we have $\Phi_{i}\left(\varphi^{\prime}\right)\left(u_{j}^{i}\right)=$ $\Phi_{i}(\varphi)\left(u_{j}^{i}\right)$. This proves that $\Phi_{i}(\varphi)=\Phi_{i}\left(\varphi^{\prime}\right)$, which implies that the isomorphism (3.8) is independent of the choice of $\varphi$.

This proves that the $\mathrm{bl}_{V}^{\mathbb{C}^{*}} U$ is independent of the embedding $U \subset V$.

Because $\mathrm{bl}_{V}^{\mathbb{C}^{*}} U$ is independent of the embedding $U \subset V$, in the following we will drop $V$ from the notation and denote by $\mathrm{bl}^{\mathbb{C}^{*}} U$ the $\mathbb{C}^{*}$-intrinsic blow up of $U$.

The affine case: Let $Y$ be an affine scheme, let $Y_{0} \subset Y$ be a closed subscheme and let $\hat{Y}$ be the formal completion of $Y$ along $Y_{0}$. We assume $\hat{Y}$ is a $\mathbb{C}^{*}$-scheme so that $(\hat{Y})^{\mathbb{C}^{*}}=Y_{0}$ as schemes.

The pair $Y_{0} \subset \hat{Y}$ is the pair studied in the formal case. Thus we can form a $\mathbb{C}^{*}$-intrinsic blow-up bl ${ }^{\mathbb{C}^{*}} \hat{Y}$. We now show that we can glue bl ${ }^{\mathbb{C}^{*}} \hat{Y}$ with $Y-Y_{0}$ to form a $\mathbb{C}^{*}$-intrinsic blow-up of $Y$.

We let $A=\Gamma\left(\mathcal{O}_{Y}\right), I \subset A$ the ideal defining $Y_{0} \subset Y$; thus $\hat{A}=\lim A / I^{n}=\Gamma\left(\mathcal{O}_{\hat{Y}}\right)$. We cover bl ${ }^{\mathbb{C}^{*}} \hat{Y}$ by $\mathbb{C}^{*}$-invariant affine $Z_{i}$; we denote $B_{i}=\Gamma\left(\mathcal{O}_{Z_{i}}\right)$, and $\xi_{i} \in B_{i}$ the element defining the exceptional divisor of $\pi_{i}: Z_{i} \rightarrow \hat{Y}$. For each $i$, we glue $Y$ and $Z_{i}$ as follows. Let $\hat{I}=\lim I / I^{n}$ be the ideal defining $Y_{0} \subset \hat{Y}$. We form the localization $\hat{A}_{\hat{I}}$ and $\left(B_{i}\right)_{\left(\xi_{i}\right)}$. Without loss of generality, we can assume that for each $i, \hat{A}_{\hat{I}}=\left(B_{i}\right)_{\left(\xi_{i}\right)}$. 
For each $i$, we form the direct sum module $B_{i} \oplus A$ and define the ring structure: $(b, a) \cdot\left(b^{\prime}, a^{\prime}\right)=\left(b b^{\prime}, a a^{\prime}\right)$. We then define $\tilde{B}_{i}$ to be the kernel

$$
\tilde{B}_{i}=\operatorname{ker}\left\{B_{i} \oplus A \longrightarrow \hat{A}_{\hat{I}}\right\}
$$

where $A \rightarrow \hat{A}_{\hat{I}}$ is the composite of the tautological $A \rightarrow \hat{A}$ with the localization homomorphism; $B_{i} \rightarrow \hat{A}_{\hat{I}}$ is the negative of the local homomophism $B_{i} \rightarrow\left(B_{i}\right)_{\left(\xi_{i}\right)}=$ $\hat{A}_{\hat{I}}$.

It is routine to check that $\operatorname{Spec} \tilde{B}_{i}$ patch to form a scheme together with a morphism

$$
\pi: \mathrm{bl} \mathbb{C}^{*} Y \longrightarrow Y
$$

it satisfies $\mathrm{bl}^{\mathbb{C}^{*}} Y \times_{Y} \hat{Y}=\mathrm{bl}^{\mathbb{C}^{*}} \hat{Y}$. The scheme $\mathrm{bl}^{\mathbb{C}^{*}} Y$ is the $\mathbb{C}^{*}$-intrinsic blow-up of $Y$ along $Y_{0}$. By the independence on the embedding proved in the formal case, it is canonically defined based on the $\mathbb{C}^{*}$-structure of $\hat{Y}$.

The scheme case: Let $Y_{0} \subset Y$ be as in the previous case except that it is no longer assumed to be affine. Let $\hat{Y}$ be the formal completion of $Y$ along $Y_{0}$. We assume $\hat{Y}$ is a $\mathbb{C}^{*}$-scheme so that $(\hat{Y})^{\mathbb{C}^{*}}=Y_{0}$.

We cover $Y$ by affine open $Y_{i} \rightarrow Y$; we let $Y_{i, 0}=Y_{i} \cap Y_{0}$, and let $\hat{Y}_{i}$ be the formal completion of $Y_{i}$ along $Y_{i, 0}$. Since $\hat{Y}_{i}=\hat{Y} \times_{Y} Y_{i}$, it is a $\mathbb{C}^{*}$-scheme. We let bl ${ }^{\mathbb{C}^{*}} Y_{i}$ be its $\mathbb{C}^{*}$-intrinsic blow up. For each pair $(i, j)$, using that $\hat{Y}_{i} \times_{Y} Y_{j}=\hat{Y}_{j} \times_{Y} Y_{i}$ as $\mathbb{C}^{*}$ schemes, we have a canonical isomorphism bl ${ }^{\mathbb{C}^{*}} Y_{i} \times{ }_{Y} Y_{j}=\mathrm{bl}^{\mathbb{C}^{*}} Y_{j} \times_{Y} Y_{i}$. This shows that we can patch $\mathrm{bl}^{\mathbb{C}^{*}} Y_{i}$ to form a scheme, which we call the $\mathbb{C}^{*}$-intrinsic blow-up of $Y$, and denote by $\mathrm{bl}^{\mathbb{C}^{*}} Y$.

The DM-stack case: We let $X$ be a DM-stack with a $\mathbb{C}^{*}$-action. We assume that the multiplication morphism $\sigma: \mathbb{C}^{*} \times X \rightarrow X$ is representable.

We let $Y$ be a scheme and $Y \rightarrow X$ be an étale morphism. We let $Y_{0}=Y \times_{X} X^{\mathbb{C}^{*}}$, and let $\hat{Y}$ be the formal completion of $Y$ along $Y_{0}$. Using that the multiplcation morphism $\sigma$ is representable, one checks that the $\mathbb{C}^{*}$-action on $Y$ induces a $\mathbb{C}^{*}$-action on $\hat{Y}$ such that $\hat{Y}^{\mathbb{C}^{*}}=Y_{0}$. Therefore, we can form the $\mathbb{C}^{*}$-intrinsic blow-up bl ${ }^{\mathbb{C}} Y$.

This construction is canonical. Let $Y^{\prime} \rightarrow X$ be another étale chart of $X$, and let bl ${ }^{\mathbb{C}^{*}} Y^{\prime}$ be the $\mathbb{C}^{*}$-intrinsic blow-up of $Y^{\prime}$. Then $Y \times_{X} Y^{\prime}$ is étale over $X$, and has its $\mathbb{C}^{*}$-intrinsic blow-up. One checks that $\mathrm{bl}^{\mathbb{C}^{*}}\left(Y \times_{X} Y^{\prime}\right)$ is canonically isomorphic to both $\mathrm{bl}^{\mathbb{C}^{*}} Y \times_{X} Y^{\prime}$ and $\mathrm{bl}^{\mathbb{C}^{*}} Y^{\prime} \times{ }_{X} Y$.

The $\mathbb{C}^{*}$-intrinsic blow-up bl ${ }^{\mathbb{C}^{*}} X$ is the stack that comes with covers bl ${ }^{\mathbb{C}^{*}} Y_{i}$ and products

$$
\mathrm{bl}^{\mathbb{C}^{*}} Y_{i} \times_{\mathrm{bl}^{\mathbb{C}^{*}} X} \mathrm{bl}^{\mathbb{C}^{*}} Y_{j}=\mathrm{bl}^{\mathbb{C}^{*}}\left(Y_{i} \times_{X} Y_{j}\right)
$$

Proposition 3.2. Let $X$ be a $\mathbb{C}^{*}$-equivariant DM-stack as stated, and let $Y_{i} \rightarrow$ $X$ be an étale atlas of $X$. Then the collection $\mathrm{bl}^{\mathbb{C}^{*}} Y_{i}$ together with the morphisms $\mathrm{bl}^{\mathbb{C}^{*}}\left(Y_{i} \times_{X} Y_{j}\right) \rightarrow \mathrm{bl}^{\mathbb{C}^{*}} Y_{i}$ and $\mathrm{bl}^{\mathbb{C}^{*}}\left(Y_{i} \times_{X} Y_{j}\right) \rightarrow \mathrm{bl}^{\mathbb{C}^{*}} Y_{j}$ form a DM-stack $\mathrm{bl}^{\mathbb{C}^{*}} X$ with a $\mathbb{C}^{*}$-equivariant projection $\pi: \mathrm{bl}^{\mathbb{C}^{*}} X \rightarrow X$. ical.

Proof. The proof follows from that the $\mathbb{C}^{*}$-intrinsic blow up construction is canon-

We call $\pi: \mathrm{bl}^{\mathbb{C}^{*}} X \rightarrow X$ the $\mathbb{C}^{*}$-intrinsic blow-up of $X$. 
3.2. Obstruction theory of $\bar{X}$-local theory. We now investigate the obstruction theory of $\bar{X}$ near the exceptional divisor of $\bar{X} \rightarrow X$. For notational simplicity, in the following we let $T=\mathbb{C}^{*}$ and call the $\mathbb{C}^{*}$-actions on $X$ and $\bar{X}$ the $T$-actions.

We begin with the local situation.

The set-up: We let $X \subset Y=\operatorname{Spec} A \llbracket x \rrbracket$, where $\operatorname{Spec} A$ is smooth and $x=$ $\left(x_{1}, \cdots, x_{m}\right)$, be a closed subscheme in a smooth affine scheme. We let $T$ acts on $Y$ so that it acts on $A$ trivially and on $x_{j}$ via $x_{j}^{\sigma}=\sigma^{l_{j}} x_{j}, l_{j} \neq 0$. We suppose $X \subset Y$ is $T$-invariant and admits a $T$-equivariant symmetric obstruction theory.

Because $X$ has a symmetric obstruction theory, by [2], the embedding $X \subset Y$ and its obstruction theory are defined by the vanishing of an almost closed 1-form

$$
\omega=\alpha+\sum_{j=1}^{m} f_{j} d x_{j} \in \Omega_{Y}, \quad \alpha \in \Omega_{A} \otimes_{A} A \llbracket x \rrbracket \quad \text { and } \quad f_{j} \in A \llbracket x \rrbracket .
$$

(Almost closed means $\left.d \omega\right|_{X}=0$.) Because the obstruction theory is $T$-equivariant, $\omega$ can be chosen to be $\mathbb{C}^{*}$-invariant. If we let $I_{X}$ be the ideal sheaf of $X \subset Y$, then the obstruction theory is given by

$$
V^{\bullet}:=\left[\mathcal{V} \stackrel{d \omega}{\longrightarrow} \mathcal{V}^{\vee}\right] \longrightarrow \mathbb{L}_{X}^{\geq-1}=\left[I_{X} /\left.I_{X}^{2} \rightarrow \Omega_{Y}\right|_{X}\right], \quad \mathcal{V}=\left.T_{Y}\right|_{X}
$$

We remark that if $\omega^{\prime}$ is another $T$-invariant almost closed 1-form defining $X \subset Y$ and its symmetric obstruction theory, then necessarily $\omega-\omega^{\prime} \in I_{X}^{2} \cdot \Omega_{Y}$.

We now turn our attention to the $T$-intrinsic blow-up $\bar{X}$ of $X$. Since $Y$ is smooth, the $T$-intrinsic blow-up $\bar{Y}$ of $Y$ is the usual blow-up of $Y$ along $Y^{T}$. Let $\bar{\pi}: \bar{Y} \rightarrow Y$ be the projection, let $\bar{E} \subset \bar{Y}$ be the exceptional divisor, and let $\xi \in \Gamma\left(\mathcal{O}_{\bar{Y}}(\bar{E})\right)$ be the defining equation of $\bar{E} \subset \bar{Y}$.

We consider the pull-back

$$
\bar{\pi}^{*} \omega=\bar{\pi}^{*} \alpha+\sum \bar{\pi}^{*} f_{j} d x_{j} \in \bar{\pi}^{*} \Omega_{\bar{Y}}
$$

Since $\omega$ is $T$-invariant and $d x_{i}$ has non-zero weights, $\xi^{-1} \bar{\pi}^{*} f_{j} d x_{j}$ are regular sections in $\bar{\pi}^{*} \Omega_{Y}^{\mathrm{mv}}(-\bar{E})$.

By definition, $\bar{X} \subset \bar{Y}$ is defined by the vanishing of $\bar{\pi}^{*} \alpha$ and $\xi^{-1} \bar{\pi}^{*} f_{j}$ 's. To put these into a compact form, we introduce $\epsilon_{j}=\bar{\pi}^{*} d x_{j}$, which span the sheaf $\bar{\pi}^{*} \Omega_{Y}^{\mathrm{mv}}$, where $\Omega_{Y}^{\mathrm{mv}}$ is the subsheaf of $\Omega_{Y}$ spanned by $d x_{j}$ 's. We let $\Omega_{Y}^{\mathrm{fix}}=\Omega_{A} \otimes_{A} A \llbracket x \rrbracket$ be the fixed part, and let $\overline{\mathcal{V}}_{-1}$ be the sheaf whose dual is

$$
\overline{\mathcal{V}}_{-1}^{\vee}:=\bar{\pi}^{*} \Omega_{Y}^{\mathrm{fix}} \oplus \bar{\pi}^{*} \Omega_{Y}^{\mathrm{mv}}(-\bar{E}) \equiv \operatorname{ker}\left\{\left.\bar{\pi}^{*} \Omega_{Y} \longrightarrow \bar{\pi}^{*}\left(\Omega_{Y}^{\mathrm{mv}}\right)\right|_{Y^{T}}\right\} .
$$

Then

$$
\bar{\omega}=\bar{\pi}^{*} \alpha+\sum \xi^{-1} \bar{\pi}^{*} f_{j} \cdot \epsilon_{j} \in \Gamma\left(\bar{Y}, \overline{\mathcal{V}}_{-1}^{\vee}\right)
$$

This way, $\bar{X}$ has the form

$$
\bar{X}=(\bar{\omega}=0) \subset \bar{Y}
$$

its obstruction theory is

$$
\bar{V}^{\bullet}:=\left[\overline{\mathcal{V}}_{-1} \stackrel{d \bar{\omega}^{\vee}}{\longrightarrow} \overline{\mathcal{V}}_{0}\right] \longrightarrow \mathbb{L}_{\bar{X}}^{\geq-1}=\left[I_{\bar{X}} /\left.I_{\bar{X}}^{2} \rightarrow \Omega_{\bar{Y}}\right|_{\bar{X}}\right], \quad \overline{\mathcal{V}}_{0}=\left.\Omega_{\bar{Y}}\right|_{\bar{X}} .
$$


For future reference, we comment that the complex $\bar{V}^{\bullet}$ can be constructed directly from the complex $V^{\bullet}$. Let $\mathcal{V}=\left.T_{Y}\right|_{X}$. Let $\pi: \bar{X} \rightarrow X$ be the projection and $E \subset \bar{X}$ be the exceptional divisor. Let $\ell$ be the tautological line bundle of the exceptional divisor $E$; namely, tautologically $\ell \subset \pi^{*} N_{Y^{T} / Y}$. Then

$$
\left.\overline{\mathcal{V}}_{0}^{\vee}\right|_{\bar{X}}=\operatorname{ker}\left\{\pi^{*} \mathcal{V} \rightarrow \pi^{*} N_{Y^{T} / Y} / \ell\right\}
$$

It is easy to describe the fixed locus $\bar{X}^{T}$ and its obstruction theory. Let $\mathcal{N}$ be the normal bundle $N_{Y^{T / Y}}$. Since $Y$ is smooth, the exceptional divisor $\bar{E}=\mathbb{P} \mathcal{N}$. To each $k \in \mathbb{Z}$, we let $\mathcal{N}_{(k)}$ be the weight $k$ piece of the $T$-decomposition of $\mathcal{N}$. Then

$$
\bar{Y}^{T}=\coprod_{k} \bar{Y}^{T, k}, \quad \bar{Y}^{T, k}=\mathbb{P}_{(k)}
$$

Accordingly,

$$
\bar{X}^{T}=\coprod_{k \in \mathbb{Z}} \bar{X}^{T, k}, \quad \bar{X}^{T, k}=\bar{X} \cap \bar{Y}^{T, k}
$$

The obstruction theory of $\bar{X}^{T}$, following [8], is given by the invariant part of the obstruction theory of $\bar{X}$. To proceed, we reindex the $x_{j}$ 's as $x_{k, j}$ indexed by $(k, j)$ so that the $T$-action is $t \cdot x_{k, j}=t^{k} x_{k, j}$. Then

$$
\omega=\alpha+\sum_{(k, j)} f_{k, j} d x_{k, j} \quad \text { and } \quad \bar{\omega}=\bar{\pi}^{*} \alpha+\sum_{(k, j)} \xi^{-1} \bar{\pi}^{*} f_{k, j} \cdot \epsilon_{k, j} .
$$

Restricting to $\bar{Y}^{T, k}$, the $T$-action on $f_{i, j}$ (resp. $\xi$ ) has weight $-i$ (resp. $k$ ), among all $\left.\xi^{-1} \bar{\pi}^{*} f_{i, j}\right|_{\bar{Y}^{T, k}}$, the $T$-invariant ones are

$$
\left.\xi^{-1} \bar{\pi}^{*} f_{-k, j}\right|_{\bar{Y}^{T, k}}, \quad 1 \leq j \leq e_{-k}=\operatorname{dim} \mathbb{P N}_{(-k)} / Y^{T}+1 .
$$

Therefore, the obstruction theory of $\bar{X}^{T, k} \subset \bar{Y}^{T, k}$ is induced by $\left.\bar{\pi}^{*} \alpha\right|_{\bar{Y}^{T, k}}$ and $e_{-k}$ additional equations. In particular,

$$
\text { vir. } \operatorname{dim} \bar{X}^{T, k}=\text { vir. } \operatorname{dim} X^{T}+\operatorname{dim} \mathbb{P N}_{(k)} / Y^{T}-e_{-k}=e_{k}-e_{-k}-1 .
$$

We get more from this description of the obstruction theory of $\bar{X}^{T, k}$. The defining equations of $\bar{X}^{T, k} \subset \bar{Y}^{T, k}$ divide into two groups. The first is

$$
\bar{\alpha}_{0}:=\left.\bar{\pi}^{*} \alpha\right|_{\bar{Y}^{T, k}}=0 .
$$

By the construction of $\bar{Y}^{T, k}, \bar{\alpha}_{0}=\left.\bar{\pi}^{*} \alpha\right|_{x_{i, j}=0} \in A$; it defines

$$
\mathbb{P}_{X} \mathcal{N}_{(k)}:=\mathbb{P}_{(k)} \times_{Y^{T}} X^{T}
$$

The second group is

$$
\bar{\beta}_{-k, j}:=\left.\xi^{-1} \bar{\pi}^{*} f_{-k, j}\right|_{\bar{Y}^{T, k}}=0, \quad 1 \leq j \leq e_{-k} .
$$

Expanding $f_{-k, j}=\sum_{J} a_{-k, j}^{J} x^{J}$, where $J$ is the usual multi-index convention and $a_{-k, j}^{J} \in A$, we see that

$$
\bar{\beta}_{-k, j}=\sum_{1 \leq i \leq e_{k}} \xi^{-1} a_{-k, j}^{-k, i} \bar{\pi}^{*} x_{-k, i}
$$


which involves weight $-k$ linear (in the $x$ variables) terms in $f_{-k, j}$. The equations $\bar{\beta}_{-k, j}=0$ defines the relative obstruction theory of $\bar{X}^{T, k} / \mathbb{P}_{X} \mathcal{N}_{(k)}$.

We now put this in the form of arrows in the derived category. Let $\pi_{(k)}: \bar{X}^{T, k} \rightarrow$ $Y$ be the tautological projection. Firstly, the fixed part is

$$
\left.\bar{V}^{\bullet}\right|_{\bar{X}^{T, k}} ^{\mathrm{fix}}=\left[\left.\left(\pi_{(k)}^{*} \Omega_{Y}^{\mathrm{fix}}\right)^{\vee} \oplus \pi_{(k)}^{*} \mathcal{N}_{(-k)}(-1) \rightarrow \Omega_{\bar{Y}^{T, k}}\right|_{\bar{X}^{T, k}}\right] .
$$

The obstruction theory of $\mathbb{P}_{X} \mathcal{N}_{(k)}$ is

$$
F_{(k)}^{\bullet}:=\left[\left.\left.\left(\bar{\pi}^{*} \Omega_{Y}^{\mathrm{fix}}\right)^{\vee}\right|_{\mathbb{P}_{X} \mathcal{N}_{(k)}} \rightarrow \Omega_{\bar{Y}^{T, k}}\right|_{\mathbb{P}_{X} \mathcal{N}_{(k)}}\right] \longrightarrow \mathbb{L}_{\mathbb{P}_{X} \mathcal{N}_{(k)}}
$$

the relative obstruction theory of $\bar{X}^{T, k} / \mathbb{P}_{X} \mathcal{N}_{(k)}$ is

$$
E_{(k)}[-1]:=\pi_{(k)}^{*} \mathcal{N}_{(-k)}(-1) \longrightarrow \mathbb{L}_{\bar{X}^{T, k} / \mathbb{P}_{X} \mathcal{N}_{(k)}}
$$

(Here the arrows in (3.20) and (3.21) are defined by $\bar{\alpha}_{0}$ and $\bar{\beta}_{-k, j}$, respectively.)

Using the explicit form of the defining equations inducing the arrows defining the obstruction theories of $\bar{X}^{T, k}, \mathbb{P}_{X} \mathcal{N}_{(k)}$ and $\bar{X}^{T, k} / \mathbb{P}_{X} \mathcal{N}_{(k)}$, we have the following commutative diagram of distinguished triangles

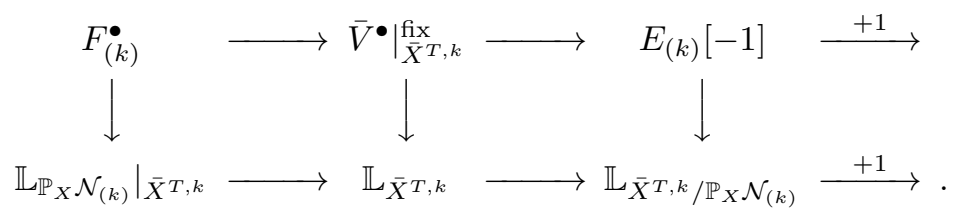

Finally, we comment that this diagram is independent of the choice of the defining equation $\omega$. (It seems to depend on the choice of the embedding $X \subset Y$.) As argued, if $\omega^{\prime}$ is another almost closed 1-form like $\omega$, then $\omega-\omega^{\prime} \in I_{X}^{2} \cdot \Omega_{Y}$. Since the left and the right vertical arrows defined by $\omega$ only use $\bar{\alpha}_{0}$ and $\bar{\beta}_{-k, j}$, to show that they are independent of the choice of $\omega$, we only need to show that

$$
\bar{\alpha}_{0}-\bar{\alpha}_{0}^{\prime} \in\left(\bar{\alpha}_{0}\right)^{2}, \quad \text { and for all } j: \quad \bar{\beta}_{-k, j}-\bar{\beta}_{-k, j}^{\prime} \in\left(\left\{\bar{\beta}_{-k, l}\right\}_{l=1}^{e_{-k}}\right)^{2} \text {. }
$$

Using the explicit forms given after (3.18) and (3.19), one sees that (3.23) is true. This shows that (3.22) is independent of the choice of $\omega$.

3.3. The virtual normal cone. The defining equation (3.14) also provides a canonical embedding of the the normal cone

$$
\left.C_{\bar{X} / \bar{Y}} \subset \overline{\mathcal{V}}_{-1}^{\vee}\right|_{\bar{X}}
$$

The $T$-action induces a homomorphism $\mathcal{O}_{Y} \rightarrow T_{Y}$; its pull-back $\mathcal{O}_{\bar{Y}} \rightarrow \bar{\pi}^{*} T_{Y}$ vanishes simply along $E$. This gives an injective homomorphism $\mathcal{O}_{\bar{Y}}(E) \hookrightarrow \bar{\pi}^{*} T_{Y}$ and its surjective dual

$$
\bar{\pi}^{*} \Omega_{Y} \longrightarrow \mathcal{O}_{\tilde{Y}}(-E)
$$

Since (3.25) factors through the moving part of $\bar{\pi}^{*} \Omega_{Y}$, using (3.12), it induces a surjective homomorphism

$$
\bar{\sigma}: \overline{\mathcal{V}}_{-1}^{\vee} \longrightarrow \mathcal{O}_{\bar{Y}}(-2 E)
$$


We let

$$
\operatorname{ker}\{\bar{\sigma}\}=\operatorname{ker}\left\{\bar{\sigma}: \overline{\mathcal{V}}_{-1}^{\vee} \rightarrow \mathcal{O}_{\bar{Y}}(-2 E)\right\} \subset \overline{\mathcal{V}}_{-1}^{\vee}
$$

LEMmA 3.3. The support of the cone $C_{\bar{X} / \bar{Y}}$ (cf. (3.24)) lies in the subbundle $\left.\operatorname{ker}\{\bar{\sigma}\}\right|_{\bar{X}}$.

The proof relies on the following criterion proved in [11, Lemma 4.5]. Let $\bar{I}$ be the ideal sheaf of $\bar{X} \subset \bar{Y}$.

Criterion: The support of $\left.C_{\bar{X} / \bar{Y}} \subset \overline{\mathcal{V}}_{-1}^{\vee}\right|_{\bar{X}}$ lies in $\operatorname{ker}\{\bar{\sigma}\}$ if the following holds: for any closed $\bar{o} \in \bar{X}$ and $\bar{\varphi}:$ Spec $k \llbracket t \rrbracket \rightarrow \bar{Y}$ with $\bar{\varphi}(0)=\bar{o}, \bar{\varphi}^{*}(\bar{\sigma} \circ \bar{\omega}) \in t \cdot \bar{\varphi}^{*} \bar{I}$.

Let $I$ be the ideal sheaf of $X \subset Y$; let $F=\sum_{i=1}^{m} l_{i} x_{i} f_{i} \in \Gamma\left(Y, \mathcal{O}_{Y}\right)$. In the following, we denote by $\left(\left\{x_{i}\right\}_{1}^{n}\right)$ the ideal $\left(x_{1}, \cdots, x_{n}\right)$.

Lemma 3.4. Let $\varphi:$ Spec $\mathbf{k} \llbracket t \rrbracket \rightarrow Y$ be any morphism. Then

$$
\varphi^{*}(F) \in t \cdot \varphi^{*} I \cdot\left(\left\{\varphi^{*} x_{i}\right\}_{1}^{n}\right) .
$$

Proof. It suffices to prove the case where $A=\mathbf{k} \llbracket u \rrbracket, u=\left(u_{1}, \cdots, u_{m}\right)$.

Let $\phi_{i}(t)=\varphi^{*} u_{i}$ and $\psi_{i}(t)=\varphi^{*} x_{i}$; let $a$ and $b$ be defined by

$$
\varphi^{*} I=\left(t^{a}\right) \quad \text { and } \quad\left(\left\{\psi_{i}(t)\right\}_{1}^{n}\right)=\left(t^{b}\right) .
$$

Then $\varphi^{*}(F) \in t \cdot \varphi^{*} I \cdot\left(\left\{\varphi^{*} x_{i}\right\}_{i}\right)$ is equivalent to $\varphi^{*} F=\sum l_{i} \psi_{i} \cdot \varphi^{*} f_{i} \in\left(t^{a+b+1}\right)$.

We introduce

$$
\Phi(t, s)=\left(\phi_{i}(t) ; \psi_{j}(t) e^{l_{j} s}\right):=\left(\left\{u_{i}(t, s)\right\}_{1}^{n} ;\left\{x_{j}(t, s)\right\}_{1}^{m}\right),
$$

which is a homomorphism $\mathbf{k} \llbracket u, x \rrbracket \rightarrow \mathbf{k} \llbracket t, s \rrbracket$. Then

$$
d x_{j}(t, s)=\psi_{j}^{\prime} e^{l_{j} s} d t+l_{j} \psi_{j} e^{l_{j} s} d s .
$$

Using that $\omega$ is $\mathbb{C}^{*}$-equivariant, we have $\left(f_{i}\right)^{\sigma}=\sigma^{-l_{i}} f_{i}$. Thus,

$$
\Phi^{*} \omega=\sum \varphi^{*} f_{i} \cdot \psi_{i}^{\prime} d t+\sum l_{i} \varphi^{*} f_{i} \cdot \psi_{i} d s .
$$

Therefore,

$$
d\left(\Phi^{*} \omega\right)=\left(\sum l_{i} \varphi^{*} f_{i} \cdot \psi_{i}\right)_{t} d t \wedge d s=\left(\varphi^{*} F\right)_{t} d t \wedge d s
$$

(We use the subscript $t$ to denote $\frac{\partial}{\partial t}$; same with $x_{k}$.) On the other hand, using $\left.d \omega=\sum_{j>k}\left(\left(f_{j}\right)_{x_{k}}-\left(f_{k}\right)_{x_{j}}\right)\right) d x_{k} \wedge d x_{j}$, we obtain

$$
d\left(\Phi^{*} \omega\right)=\Phi^{*} d \omega=\sum_{j>k}\left(\Phi^{*}\left(f_{j}\right)_{x_{k}}-\Phi^{*}\left(f_{k}\right)_{x_{j}}\right) d x_{k}(t, s) \wedge d x_{j}(t, s) .
$$

Using (3.28), we calculate

$$
d x_{i}(t, s) \wedge d x_{j}(t, s)=e^{\left(l_{i}+l_{j}\right) s}\left(l_{j} \psi_{i}^{\prime} \cdot \psi_{j}-l_{i} \psi_{i} \cdot \psi_{j}^{\prime}\right) d t \wedge d s .
$$

Applying the $\mathbb{C}^{*}$-equivariance, (3.30) reduces to

$$
d\left(\Phi^{*} \omega\right)=\sum_{j>k}\left(\varphi^{*}\left(f_{j}\right)_{x_{k}}-\varphi^{*}\left(f_{k}\right)_{x_{j}}\right)\left(l_{j} \psi_{i}^{\prime} \cdot \psi_{j}-l_{i} \psi_{i} \cdot \psi_{j}^{\prime}\right) d t \wedge d s
$$


Since $\omega$ is almost closed, $\left(f_{j}\right)_{x_{k}}-\left(f_{k}\right)_{x_{j}} \in I$ for all $j, k$. We then use (3.27) to conclude that

$$
\left(\varphi^{*}\left(f_{j}\right)_{x_{k}}-\varphi^{*}\left(f_{k}\right)_{x_{j}}\right)\left(l_{j} \psi_{i}^{\prime} \cdot \psi_{j}-l_{i} \psi_{i} \cdot \psi_{j}^{\prime}\right) \in\left(t^{a+2 b-1}\right) .
$$

Thus equating (3.29) and (3.31), we conclude $\left(\varphi^{*} F\right)_{t} \in\left(t^{a+2 b-1}\right)$. Since $\varphi^{*} F(0)=0$, $\varphi^{*} F \in\left(t^{a+2 b}\right)$. Since $b \geq 1$, this proves $\varphi^{*} F \in\left(t^{a+b+1}\right)$. This proves the Lemma.

Proof of Lemma 3.3. As before, we only need to prove the case where $A=\mathbf{k} \llbracket u \rrbracket$, $u=\left(u_{1}, \cdots, u_{m}\right)$. We verify the criterion. Let $\bar{\varphi}: \operatorname{Spec} \mathbf{k} \llbracket t \rrbracket \rightarrow \bar{Y}, \bar{\varphi}(0)=\bar{o} \in \bar{X}$ be any morphism. We will show that $\bar{\varphi}^{*}(\bar{\sigma} \circ \bar{\omega}) \in t \cdot \bar{\varphi}^{*} \bar{I}$. Clearly, we only need to check the case where $\bar{o} \in E$.

For the moment, we assume that $\bar{\varphi}$ does not factor through $E \subset \bar{Y}$. Since $\bar{Y}$ is the blow-up of $Y$ along $x_{1}=\cdots=x_{m}=0$, canonically $\bar{Y} \subset Y \times \mathbb{P}^{m-1}$, and we can choose homogeneous coordinates of $\mathbb{P}^{m-1}$ so that $\bar{Y}$ is defined by $\frac{x_{i}}{w_{i}}=\frac{x_{j}}{w_{j}}$, for all $i \neq j$. By reordering the indices of $x_{i}$, we can assume $\bar{o} \in\left\{w_{1} \neq 0\right\}$.

Let $\varphi=\bar{\pi} \circ \bar{\varphi}:$ Spec $\llbracket t \rrbracket \rightarrow Y$. Like before, we denote $\phi_{i}(t)=\varphi^{*} u_{i}$ and $\psi_{j}(t)=$ $\varphi^{*} x_{j}$. Since $\varphi$ does not factor through $E, \psi_{1}(t) \neq 0 ; \bar{o} \in\left\{w_{1} \neq 0\right\}$ implies that as ideals,

$$
\left(t^{b}\right)=\left(\left\{\psi_{i}(t)\right\}_{1}^{n}\right)=\left(\psi_{1}(t)\right) .
$$

Then, following the definition, we have

$$
\bar{\varphi}^{*} \bar{I}=\left(\left\{\varphi^{*} g_{i}\right\}_{1}^{n},\left\{\psi_{1}(t)^{-1} \varphi^{*} f_{j}\right\}_{1}^{m}\right),
$$

and

$$
\bar{\varphi}^{*}(\bar{\sigma} \circ \bar{\omega})=\psi_{1}(t)^{-1} \sum l_{i} \varphi^{*} x_{i} \cdot \psi_{1}(t)^{-1} \varphi^{*} f_{i}=\psi_{1}(t)^{-2} \sum l_{i} \varphi^{*} x_{i} \cdot \varphi^{*} f_{i} .
$$

By the definition of $a$, we also have $\left(\psi_{1}(t)^{-1} \cdot t^{a}\right) \subset \bar{\varphi}^{*} \bar{I}$; by Lemma 3.4 and (3.32), we have

$$
\sum l_{i} \varphi^{*} x_{i} \cdot \varphi^{*} f_{i} \in t\left(\psi_{1}(t) \cdot t^{a}\right)
$$

This implies $\bar{\varphi}^{*}(\bar{\sigma} \circ \bar{\omega}) \in t \cdot \bar{\varphi}^{*} \bar{I}$.

It remains to verify the case when $\bar{\phi}$ factors through $E \subset \bar{Y}$. In this case, we can find $\bar{\varphi}_{1}$ so that $\bar{\varphi}-\bar{\varphi}_{1} \in\left(t^{k}\right)$ for a sufficiently large $k$ and $\bar{\varphi}_{1}$ does not factors through $E$. Then by what was proved, $\bar{\varphi}_{1}^{*}(\bar{\sigma} \circ \bar{\omega}) \in t \cdot \bar{\varphi}_{1}^{*} \bar{I}$. Since $k$ is sufficiently large, we conclude $\bar{\varphi}^{*}(\bar{\sigma} \circ \bar{\omega}) \in t \cdot \bar{\varphi}^{*} \bar{I}$. This proves the Lemma.

3.4. The obstruction theory of $\bar{X}$-global theory. We begin with the following situation.

We suppose there is an étale affine altas $X_{\alpha} \rightarrow X$, and two-term complexes of locally free sheaves $V_{\alpha}^{\bullet} \rightarrow \mathbb{L}_{X_{\alpha}}^{\geq-1}$ giving the symmetric obstruction theories of $X_{\alpha}$. (We will ignore the compatibility condition for the moment.)

We let $U_{\alpha}$ (resp. $\hat{X}$ ) be the formal completion of $X_{\alpha}$ (resp. $X$ ) along $X_{\alpha} \times_{X} X^{T}$ (resp. $X^{T}$ ). Since the $T$-action on $X$ is representable, each $U_{\alpha}$ is a $T$-scheme and the tautological $U_{\alpha} \rightarrow \hat{X}$ is $T$-equivariant.

We then pick smooth affine $T$-schemes $Y_{\alpha}$ and $T$-equivariant embeddings $U_{\alpha} \rightarrow$ $Y_{\alpha}$. We let $\hat{V}_{\alpha}^{\bullet}=\left[\hat{\mathcal{V}}_{\alpha} \rightarrow \hat{\mathcal{V}}_{\alpha}^{\vee}\right] \rightarrow \mathbb{L}_{\bar{U}_{\alpha}^{-1}}^{\geq}$be the pull-back to $U_{\alpha}$ of $V_{\alpha}^{\bullet} \rightarrow \mathbb{L}_{\bar{X}_{\alpha}}^{\geq}$. Since $V_{\alpha}^{\bullet} \rightarrow \mathbb{L}_{X_{\alpha}}^{\geq-1}$ are symmetric obstruction theories, by [2] and that $T$ is reductive, 
there are $T$-invariant almost closed 1-forms $\omega_{\alpha} \in \Gamma\left(\Omega_{Y_{\alpha}}\right)$ so that $U_{\alpha}=\left(\omega_{\alpha}=0\right)$, and for $\mathcal{W}_{\alpha}=\left.T_{Y_{\alpha}}\right|_{U_{\alpha}}$ there are quasi-isomorphisms that make the following squares commutative

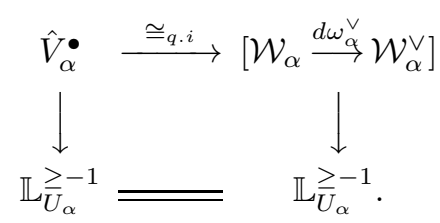

Here the right vertical arrow is the obstruction theory of $U_{\alpha}$ induced from $\omega_{\alpha}=0$. By shrinking $X_{\alpha}$ and altering the dimensions of $Y_{\alpha}$ if necessary, we can assume the (top line) quasi-isomorphism is an isomorphism.

We let $\bar{U}_{\alpha} \subset \bar{Y}_{\alpha}$ be the pair of $T$-intrinsic blow-up of $U_{\alpha} \subset Y_{\alpha}$, let $\pi_{\alpha}: \bar{U}_{\alpha} \rightarrow U_{\alpha}$ be the projection. Following the convention in the previous subsection, we let $\bar{E}_{\alpha} \subset \bar{Y}_{\alpha}$ be the exceptional divisor, let $N_{\alpha}=N_{Y_{\alpha}^{T} / Y_{\alpha}}=\left.\mathcal{W}_{\alpha}\right|_{Y_{\alpha}^{T}}$ and let $\ell_{\alpha} \subset \pi_{\alpha}^{*} N_{\alpha}$ be the tautological subline bundle. We form

$$
\overline{\mathcal{W}}_{0, \alpha}=\operatorname{ker}\left\{\pi_{\alpha}^{*} \mathcal{W}_{\alpha} \rightarrow\left(\pi_{\alpha}^{*} N_{\alpha}\right) / \ell_{\alpha}\right\}^{\vee} \quad \text { and } \quad \overline{\mathcal{W}}_{-1, \alpha}=\operatorname{ker}\left\{\pi_{\alpha}^{*} \mathcal{W}_{\alpha}^{\vee} \rightarrow \pi_{\alpha}^{*}\left(\left.\mathcal{W}_{\alpha}^{\vee}\right|_{Y_{\alpha}^{T}} ^{\mathrm{mv}}\right)\right\}^{\vee}
$$

(Note $\overline{\mathcal{W}}_{0, \alpha}=\left.T_{\bar{Y}_{\alpha}}\right|_{\bar{U}_{\alpha}}$.) The induced perfect obstruction theory of $\bar{U}_{\alpha}$ is given by

$$
\left[\overline{\mathcal{W}}_{-1, \alpha} \stackrel{d \bar{\omega}_{\alpha}^{\vee}}{\longrightarrow} \overline{\mathcal{W}}_{0, \alpha}\right] \longrightarrow \mathbb{L}_{\bar{U}_{\alpha}}^{\geq-1}
$$

Using the isomorphisms at the top line of (3.34), we can glue (3.35) with the restriction to $\bar{X}_{\alpha}-\bar{E}_{\alpha}$ of the top line of (3.34) to obtain a new complex with an arrow over $\bar{X}_{\alpha}$ :

$$
\bar{V}_{\alpha}^{\bullet}=\left[\overline{\mathcal{V}}_{-1, \alpha} \rightarrow \overline{\mathcal{V}}_{0, \alpha}\right] \longrightarrow \mathbb{L}_{\bar{X}_{\alpha}}^{\geq-1}
$$

This arrow gives the induced perfect obstruction theory of $\bar{X}_{\alpha}$.

In order that the collection $V_{\alpha}^{\bullet} \rightarrow \mathbb{L}_{\bar{X}_{\alpha}}^{\geq-1}$ gives the symmetric obstruction theory of $X$, it must satisfy certain compatibility condition. Since the construction of the induced perfect obstruction theory on $\bar{X}_{\alpha}$ is canonical, its compatibility largely follows from the compatibility of $V_{\alpha}^{\bullet} \rightarrow \mathbb{L}_{X_{\alpha}}^{\geq-1}$. For instance, if $V_{\alpha}^{\bullet} \rightarrow \mathbb{L}_{X_{\alpha}}^{\geq-1}$ are restrictions of the symmetric obstruction theory $V^{\bullet}=\left[\mathcal{V} \rightarrow \mathcal{V}^{\vee}\right] \rightarrow \mathbb{L}_{X}^{\geq-1}$ of $X$, then $\bar{V}_{\alpha}^{\bullet}$ constructed in (3.36) patch to form a global complex $\bar{V}^{\bullet}$ on $\bar{X}$. However, the arrows $\left.\bar{V}^{\bullet}\right|_{\bar{X}_{\alpha}} \rightarrow$ $\mathbb{L}_{\bar{X}_{\alpha}}^{\geq-1}$ given in (3.36) may not coincide, the reason being that the presentation $\mathbb{L}_{\bar{X}_{\alpha}}^{\geq-1}$ uses the auxiliary embeddings $U_{\alpha} \rightarrow Y_{\alpha}$.

We comment that this construction yields a local symmetric obstruction theory to be formulated in [3].

Proposition 3.5. Let $X$ be a T-equivariant DM-stack with a T-equivariant symmetric obstruction theory. Suppose that $X$ has a T-equivariant embedding $X \rightarrow Y$ into a smooth DM-stack. Then the T-intrinsic blow-up $\bar{X}$ has an induced perfect obstruction theory.

Proof. Let $X \rightarrow Y$ be the $T$-equivariant embedding into a smooth DM-stack. By Lemma 2.13, $X$ has a $T$-equivariant symmetric obstruction theory

$$
V^{\bullet}=\left[\mathcal{V} \rightarrow \mathcal{V}^{\vee}\right] \rightarrow \mathbb{L}_{\bar{X}}^{\geq-1}=\left[I_{X} /\left.I_{X}^{2} \rightarrow \Omega_{Y}\right|_{X}\right], \quad \mathcal{V}=\left.T_{Y}\right|_{X}
$$


We let $\bar{X} \subset \bar{Y}$ be the $T$-intrinsic blow-up of the pair $X \subset Y$, let $\pi: \bar{X} \rightarrow X$ be the projection, and let $\bar{E} \subset \bar{Y}$ be the exceptional divisor. We let $\overline{\mathcal{V}}_{-1}$ and $\overline{\mathcal{V}}_{0}$ be the two locally free sheaves on $\bar{X}$ given in (3.12) and (3.16).

We cover $X$ by affine étale atlas $X_{\alpha} \rightarrow X$. Let $\bar{X}_{\alpha}$ be the $\mathbb{C}^{*}$-intrinsic blow-up. Over $\bar{X}_{\alpha}$, the pull-back of $\overline{\mathcal{V}}_{-1}$ and $\overline{\mathcal{V}}_{0}$ are the sheaves $\overline{\mathcal{V}}_{-1, \alpha}$ and $\overline{\mathcal{V}}_{0, \alpha}$ mentioned in (3.36). Let $\bar{V}_{\alpha}^{\bullet} \rightarrow \mathbb{L}_{\bar{X}_{\alpha}}^{\geq-1}$ be as in (3.36).

As argued, the arrows in (3.36) are independent of the choice of an almost closed 1-form $\omega_{\alpha}$; thus the collection $\bar{V}_{\alpha} \rightarrow \mathbb{L}_{\bar{X}_{\alpha}}^{\geq-1}$ patches to form a global complex and an arrow

$$
\bar{V}^{\bullet}=\left[\overline{\mathcal{V}}_{-1} \rightarrow \overline{\mathcal{V}}_{0}\right] \longrightarrow \mathbb{L}_{\bar{X}}^{\geq-1}=\left[I_{\bar{X}} /\left.I_{\bar{X}}^{2} \rightarrow \Omega_{\bar{Y}}\right|_{\bar{X}}\right]
$$

Also, because the construction is canonical, it is $T$-equivariant. This proves the Proposition.

Let $\bar{X}^{T, k} \subset \bar{Y}^{T, k}\left(\subset \bar{Y}^{T}\right)$ be the decomposition of the fixed locus shown in (3.17). (Because the inclusion $X \subset Y$ is a global $T$-embedding, the local constructions patch to form a global decomposition.) Let $N_{(k)}$ be the weight $k$ component of $N_{Y^{T} / Y}$, and let $\mathbb{P}_{X} N_{(k)}=\mathbb{P} N_{(k)} \times_{\bar{Y}^{T}} \bar{X}^{T}$ be as defined in the previous subsection. We form a complex $F_{(k)}^{\bullet}$ and a sheaf $E_{(k)}$, as in (3.20) and (3.21). By the remark after the diagram (3.22), we have the following Lemma.

LEMMA 3.6. The constructions preceding (3.22) patch to give an obstruction theory of $\mathbb{P}_{X} N_{(k)}$ and a relative obstruction theory of $\bar{X}^{T, k} / \mathbb{P}_{X} N_{(k)}$. They fit into the commutative diagram of distinguished triangles shown in (3.22).

By Lemma 3.3, we obtain the following.

Corollary 3.7. Let the situation be as in Proposition 3.5. Then the virtual normal cone $C_{\bar{X}} \subset \overline{\mathcal{V}}_{-1}^{\vee}$ of $\bar{X}$ lies entirely in the kernel of $\overline{\mathcal{V}}_{-1}^{\vee} \rightarrow \mathcal{O}_{\bar{X}}(-2 \bar{E})$.

4. The master space. In this section we define the master space of a simple flip

$$
M_{ \pm}=\left[X_{ \pm} / \mathbb{C}^{*}\right] \subset \mathcal{M}=\left[X / \mathbb{C}^{*}\right]
$$

and prove that it is a proper separated DM-stack. We also define and study the master space of the $\mathbb{C}^{*}$-intrinsic blow-up $\bar{X}$. This master space will be the main tool for our wall crossing formula in the subsequent section.

4.1. Master space. Let $M_{ \pm}=\left[X_{ \pm} / T\right] \subset \mathcal{M}$ be the simple flip defined in Definition 2.2. We consider $X \times \mathbb{P}^{1}$ with the $T$-action

$$
\sigma \cdot\left(w,\left[s_{0}, s_{1}\right]\right)=\left(\sigma \cdot w,\left[\sigma \cdot s_{0}, s_{1}\right]\right)
$$

We pick a $T$-invariant open subset

$$
\left(X \times \mathbb{P}^{1}\right)^{s}=X \times \mathbb{P}^{1}-\Sigma_{-} \times\{0\}-\Sigma_{+} \times\{\infty\},
$$

where $0=[0,1]$ and $\infty=[1,0]$; we then form the quotient

$$
Z=\left(X \times \mathbb{P}^{1}\right)^{s} / T \text {. }
$$

Obviously, $\left(X \times \mathbb{P}^{1}\right)^{s}$ contains both $X_{+} \times\{0\}$ and $X_{-} \times\{\infty\}$ as closed substacks. 
Definition 4.1. We call $Z$ the master space for $\mathcal{M}$.

We will see below that $Z$ is a proper separated $\mathbb{C}^{*}$-equivariant DM-stack.

ExAmple 4.2. For the $X$ in Example 2.4, the master space $Z$ is simply the blow-up of $\mathbb{P} V$ along $\mathbb{P} V_{+} \cup \mathbb{P} V_{-}$.

We intend to show that the master space $Z$ is proper. In the discussion below, we will use $R$ to denote a discrete valuation ring over $\mathbb{C}$ with fractional field $K$; denote by $\zeta$ its uniformizing parameter, and denote by $\xi$ and $\xi_{0}$ its generic and closed points. Also, for an $f: \operatorname{Spec} K \rightarrow X$ and $g:$ Spec $K \rightarrow T$, we denote by $g \cdot f$ the composite

$$
\operatorname{Spec} K \stackrel{(g, f)}{\longrightarrow} T \times X \longrightarrow X
$$

where the second arrow is the group action morphism.

LEMMA 4.3. The master space $Z$ is a proper separated DM-stack.

Proof. Let $\mathcal{Z}=\left(X \times \mathbb{P}^{1}\right)^{s}$ so that $Z=[\mathcal{Z} / T]$. It is direct to check that the stabilizer of any closed $z \in \mathcal{Z}$ is finite. Also, all $T$-orbits of $\mathcal{Z}$ are closed orbits. Thus $Z$ is a separated DM-stack.

It remains to prove that $Z$ is proper. Let $R$ be a discrete valuation ring over $\mathbb{C}$ with field of fractions $K$, and let $f: \operatorname{Spec} K \rightarrow \mathcal{Z}$ be a morphism. We need to show that after a finite extension $\tilde{R} \supset R$ with $\tilde{K}$ its field of fractions, there is a morphism $g: \operatorname{Spec} \tilde{K} \rightarrow T$ so that $g \cdot f: \operatorname{Spec} \tilde{K} \rightarrow \mathcal{Z}$ extends to $(g \cdot f)^{e x}: \operatorname{Spec} \tilde{R} \rightarrow \mathcal{Z}$.

First, note that $\mathcal{Z}$ decomposes into the disjoint union

$$
\mathcal{Z}=X_{0} \times T \sqcup \Sigma_{-}^{\circ} \times T \sqcup \Sigma_{+}^{\circ} \times T \sqcup X_{+} \times\{0\} \sqcup X_{-} \times\{\infty\} \sqcup X^{T} \times T .
$$

Using $\mathcal{Z} \subset X \times \mathbb{P}^{1}$, we can write

$$
f=\left(f_{1}, f_{2}\right): \operatorname{Spec} K \longrightarrow X \times \mathbb{P}^{1} .
$$

Let $\xi$ and $\xi_{0}$ be the generic and closed point of $\operatorname{Spec} R$.

We first consider the case $f(\xi) \in X_{+} \times\{0\}$. Since by assumption the quotient $\left[X_{+} \times\{0\} / T\right]=\left[X_{+} / T\right]=M_{+}$is proper, the extension $(g \cdot f)^{e x}$ does exist. The case $f(\xi) \in X_{-} \times\{\infty\}$ is similar. For the same reason, if $f(\xi) \in X^{T} \times T$, because $X^{T}$ is proper, the extension also exists.

We now suppose $f(\xi) \in X_{0} \times T$. Because $M_{+}=\left[X_{+} / T\right]$ is proper, after a finite extension $\tilde{R}$ of $R$, we can find a morphism $g_{+}:$Spec $\tilde{K} \rightarrow T$ so that $g_{+} \cdot f: \operatorname{Spec} \tilde{K} \rightarrow$ $X_{0} \times T$ extends to $\left(g_{+} \cdot f\right)^{\text {ex }}: \operatorname{Spec} \tilde{R} \rightarrow X_{+} \times \mathbb{P}^{1}$. By the same reason, after replacing $\tilde{R}$ by a finite extension, still denoted by $\tilde{R}$, we can find $g_{-}: \operatorname{Spec} \tilde{K} \rightarrow T$ so that $g_{-} \cdot f$ extends to $\left(g_{-} \cdot f\right)^{e x}:$ Spec $\tilde{R} \rightarrow X_{-} \times \mathbb{P}^{1}$. Let $g_{+}^{e x}$ and $g_{-}^{e x}: \operatorname{Spec} \tilde{R} \rightarrow \mathbb{P}^{1}$ be the extensions of $g_{+}$and $g_{-}$. In case $g_{+}^{e x}\left(\tilde{\xi}_{0}\right) \neq \infty$ or $g_{-}^{e x}\left(\tilde{\xi}_{0}\right) \neq 0$, then either $\left(g_{+} \cdot f\right)^{e x}$ or $\left(g_{-} \cdot f\right)^{e x}$ maps to $\mathcal{Z}$ and we are done. Suppose not. Let $g: \operatorname{Spec} \tilde{K} \rightarrow T$ be defined via $g_{+} \cdot g=g_{-}$, which gives $g \cdot\left(g_{+} \cdot f\right)=g_{-} \cdot f$. Because $g_{+}^{e x}\left(\tilde{\xi}_{0}\right)=\infty$ or $g_{-}^{e x}\left(\tilde{\xi}_{0}\right)=0$, we must have $g^{*}(t)=\alpha \cdot \zeta^{a}$ with $a>0$. Therefore by Lemma 2.10 (a), after possibly another finite extension $R \subset \tilde{R}$, we can find $g^{\prime}:$ Spec $\tilde{K} \rightarrow T$ such that $\left(g^{\prime}\right)^{*}(t)=\zeta^{a^{\prime}}$ with $0<a^{\prime}<a$ and that $\left(g^{\prime} \cdot g_{+} \cdot f\right)^{e x}\left(\tilde{\xi}_{0}\right) \in X^{T} \times T \subset \mathcal{Z}$.

Finally, we consider the case $f: \operatorname{Spec} K \rightarrow \Sigma_{+}^{\circ} \times T$. (The case to $\Sigma_{-}^{\circ} \times T$ is similar.) Since $\Sigma_{+}^{\circ} \subset X_{+}$is closed and $\left[X_{+} / T\right]$ is proper, $\left[\Sigma_{+}^{\circ} / T\right]$ is proper. Thus there is a $g:$ Spec $\tilde{K} \rightarrow T$, for a finite extension $\tilde{R} \supset R$, such that $g \cdot f$ extends to $(g \cdot f)^{e x}: \operatorname{Spec} \tilde{R} \rightarrow \Sigma_{+}^{\circ} \times \mathbb{P}^{1}$. In case $(g \cdot f)^{e x}\left(\tilde{\xi}_{0}\right) \in \Sigma_{+}^{\circ} \times \mathbb{C}$, we are done. Otherwise, 
$(g \cdot f)^{*}(t)=\alpha \cdot \zeta^{a}$ with $a<0$. Then we let $g^{\prime}: \operatorname{Spec} \tilde{K} \rightarrow T$ be so that $g^{\prime *}(t)=\alpha$. By Lemma 2.10 (b), possibly after passing through a new finite extension $\tilde{R}$, the extension $\left(g^{\prime} \cdot f\right)^{e x}:$ Spec $\tilde{K} \rightarrow \Sigma_{+} \times T$ exists. This settles the case.

Combining these, we conclude that the quotient $[Z / T]$ is a proper separated DMstack.

Let $H=\mathbb{C}^{*}$ act on $X \times \mathbb{P}^{1}$ by

$$
\left(w,\left[a_{0}, a_{1}\right]\right)^{t}=\left(w,\left[a_{0}, t a_{1}\right]\right) .
$$

Obviously the action of $H$ commutes with the action of $T$ on $X \times \mathbb{P}^{1}$ and hence $Z$ admits an induced action of $H$. The following lemma is straightforward.

Lemma 4.4. The $H$-fixed point substack $Z^{H} \subset Z$ is the disjoint union of

$$
M_{+}=X_{+} / T, \quad M_{-}=X_{-} / T, \text { and } X^{T} \text {. }
$$

For the obstruction theory, we have

Lemma 4.5. If the quotient stack $\mathcal{M}=[X / T]$ admits a perfect obstruction theory, then the master space $Z$ has an induced $H$-equivariant perfect obstruction theory.

Proof. By definition, $X$ comes with a $T$-equivariant perfect obstruction theory. The perfect obstruction theory of $X$ lifts to a $T \times H$-equivariant perfect obstruction theory of $X \times \mathbb{P}^{1}$, which restricts to the open substack $\left(X \times \mathbb{P}^{1}\right)^{s}$. Since $T$ acts with only finite stabilizers, the quotient $Z$, as we saw earlier for $M_{ \pm}$, has an induced $H$-equivariant perfect obstruction theory. This proves the lemma.

It follows from the proof that vir. $\operatorname{dim} Z=$ vir. $\operatorname{dim} X=$ vir. $\operatorname{dim} M_{ \pm}=0$.

4.2. Master space for $\bar{X}$. By the same construction as above, we can define the master space $\bar{Z}$ for the $\mathbb{C}^{*}$-intrinsic blow-up $\bar{X}$. We will show that $M_{ \pm}$is a subset of the fixed points of $\bar{X}$.

Consider $\bar{X} \times \mathbb{P}^{1}$ with $T$-action

$$
\left(w,\left[s_{0}, s_{1}\right]\right)^{\sigma}=\left(w^{\sigma},\left[\sigma \cdot s_{0}, s_{1}\right]\right)
$$

and let

$$
\left(\bar{X} \times \mathbb{P}^{1}\right)^{s}:=\bar{X} \times \mathbb{P}^{1}-\left(\Sigma_{-}^{\circ} \cup \bar{E}\right) \times\{0\}-\left(\Sigma_{+}^{\circ} \cup \bar{E}\right) \times\{\infty\}
$$

where $\bar{E}$ is the inverse image of $X^{T}$ in $\bar{X}$. The master space $\bar{Z}$ is defined as the quotient of the open stack $\left(\bar{X} \times \mathbb{P}^{1}\right)^{s}$ by the action of $T$. The same argument as in the proof of Lemma 4.3 proves that $\bar{Z}$ is a proper separated DM-stack. By construction, $\bar{Z}$ has a partition

$$
\bar{Z}=\bar{X} \sqcup M_{+} \sqcup M_{-},
$$

where $\bar{X}$ is identified with the quotient of $\bar{X} \times\left(\mathbb{P}^{1}-\{0, \infty\}\right)$ by $T$.

The action of $H=\mathbb{C}^{*}$ on $\bar{X} \times \mathbb{P}^{1}$ by $\left(w,\left[a_{0}, a_{1}\right]\right)^{\sigma}=\left(w,\left[a_{0}, \sigma \cdot a_{1}\right]\right)$ induces an $H$-action on $\bar{Z}$. It is straightforward to check that the $H$-fixed point set in $\bar{Z}$ is the (disjoint) union

$$
\bar{Z}^{H}=\bar{X}^{T} \sqcup M_{+} \sqcup M_{-} .
$$

When $\mathcal{M}=[X / T]$ is equipped with a symmetric obstruction theory, Proposition 3.5 gives us a perfect obstruction theory of $\bar{X}$ of virtual dimension 0 . By pulling back this obstruction theory, $\left(\bar{X} \times \mathbb{P}^{1}\right)^{s}$ is equipped with a perfect obstruction theory of virtual dimension 1 ; it then induces a perfect obstruction theory on the master space $\bar{Z}$ of virtual dimension 0 . 
5. A wall crossing formula for symmetric obstruction theories. In this section, we prove a wall crossing formula for simple flips with symmetric obstruction theory. Let $M_{ \pm}=\left[X_{ \pm} / T\right] \subset \mathcal{M}$ be a simple flip. We assume that $X$ embeds $T$ equivariantly in a smooth DM-stack $Y$.

We consider the projections

$$
\bar{X} \stackrel{q_{-}}{\longleftarrow}\left(\bar{X} \times \mathbb{P}^{1}\right)^{s} \stackrel{q_{+}}{\longrightarrow} \bar{Z} .
$$

We use the notation of Proposition 3.5 and Corollary 3.7. The pull-back $q_{-}^{*} \bar{V}^{\bullet}$ descends to the quotient stack $\bar{Z}$; we denote the descent by $\left[\mathcal{V}_{-1} \rightarrow \mathcal{V}_{0}\right]$. Similarly, $q_{-}^{*} \mathcal{O}_{\bar{X}}(-2 \bar{E})$ descends to an invertible sheaf on $\bar{Z}$, denoted by $\mathcal{O}_{\bar{Z}}(-2 \bar{E})$.

Let $C_{\bar{Z}} \subset \mathcal{V}_{-1}^{\vee}$ be the virtual normal cone cycle of $\bar{Z}$, where by abuse of notation we denote by $\mathcal{V}_{-1}^{\vee}$ also the vector bundle associated to $\mathcal{V}_{-1}^{\vee}$. By Corollary 3.7, the cycle $C_{\bar{Z}}$ lies in the kernel bundle

$$
\mathcal{V}_{-1, \text { red }}^{\vee}=\operatorname{ker}\left\{\mathcal{V}_{-1}^{\vee} \longrightarrow \mathcal{O}_{\bar{Z}}(-2 \bar{E})\right\}
$$

We let $[\bar{Z}]^{\text {vir }}=0_{\mathcal{V}_{-1}^{\vee}}^{v^{\vee}}\left[C_{\bar{Z}}\right]$ and $[\bar{Z}]_{\text {red }}^{\text {vir }}=0_{\mathcal{V}_{-1, \text { red }}^{\vee}}^{{ }^{\vee}}\left[C_{\bar{Z}}\right]$, as $H$-equivariant cycles. Thus

$$
[\bar{Z}]^{\text {vir }}=[\bar{Z}]_{\text {red }}^{\operatorname{vir}} \cap c_{1}\left(\mathcal{O}_{\bar{Z}}(-2 \bar{E})\right) \text {. }
$$

Applying the virtual localization theorem [8] to $\bar{Z}$, and using (4.2), we obtain

$$
[\bar{Z}]^{\mathrm{vir}}=\frac{\left[\bar{X}^{T}\right]^{\mathrm{vir}}}{e\left(N^{\mathrm{vir}}\right)}-\left[M_{+}\right]^{\mathrm{vir}}-\left[M_{-}\right]^{\mathrm{vir}} .
$$

After dividing both sides by $c_{1}\left(\mathcal{O}_{\bar{X}}(-2 \bar{E})\right)$, we obtain

$$
[\bar{Z}]_{\mathrm{red}}^{\mathrm{vir}}=\frac{\left[\bar{X}^{T}\right]^{\mathrm{vir}}}{e\left(N^{\mathrm{vir}}\right) c_{1}\left(\mathcal{O}_{\bar{Z}}(-2 \bar{E})\right)}+\left[M_{+}\right]^{\mathrm{vir}} /(-t)+\left[M_{-}\right]^{\mathrm{vir}} / t
$$

where $t \in A_{\mathbb{C}^{*}}^{1}(p t)$ is the standard generator of $A_{\mathbb{C}^{*}}^{*}(p t)$. Taking the residue at $t=0$, the left hand side vanishes because $[\bar{Z}]_{\text {red }}^{\text {vir }}$, as an equivariant class, has no negative degree terms in $t$. We thus obtain

$$
\operatorname{deg}\left[M_{+}\right]^{\text {vir }}-\operatorname{deg}\left[M_{-}\right]^{\text {vir }}=\operatorname{res}_{t=0} \frac{\left[\bar{X}^{T}\right]^{\text {vir }}}{e\left(N^{\text {vir }}\right) c_{1}\left(\mathcal{O}_{\bar{Z}}(-2 E)\right)} .
$$

To proceed, we need a description of the cycle $\left[\bar{X}^{T}\right]^{\text {vir }}$. We keep the notation introduced after (3.16). Namely, $\mathcal{N}=N_{Y^{T} / Y}, \mathcal{N}_{(k)}$ is the weight $k$ piece of $\mathcal{N}$, $\bar{Y}^{T, k}=\mathbb{P} \mathcal{N}_{(k)}$, and $\bar{X}^{T}=\coprod_{k \neq 0} \bar{X}^{T, k}$, where $\bar{X}^{T, k}=\bar{Y}^{T, k} \cap \bar{X}$.

As argued in [8], the obstruction theory of $X^{T}$ is given by $\left[\mathcal{V} \rightarrow \mathcal{V}^{\vee}\right]^{T} \rightarrow \mathbb{L}_{X^{T}}^{\geq-1}$ and has virtual dimension zero. Thus

$$
\left[X^{T}\right]^{\mathrm{vir}}=\sum_{i=1}^{r} a_{i}\left[p_{i}\right], \quad a_{i} \in \mathbb{Q}, p_{i} \in X^{T} \text { closed. }
$$

Let $\bar{\pi}_{(k)}: \mathbb{P} \mathcal{N}_{(k)} \rightarrow Y^{T}$ be the projection.

LEMMA 5.1. With the above notation, we have

$$
\left[\bar{X}^{T}\right]^{\mathrm{vir}}=\sum_{1 \leq i \leq r, k \neq 0} a_{i} \cdot e\left(\bar{\pi}_{(k)}^{*} \mathcal{N}_{(-k)}^{\vee}(1)\right) \cap\left[\mathbb{P}_{(k)} \times_{Y^{T}} p_{i}\right]
$$


Proof. Using diagram (3.22) and the explicit form $E_{(k)}=\left.\bar{\pi}_{(k)}^{*} \mathcal{N}_{(-k)}(-1)\right|_{\bar{X}^{T, k}}$, applying the main result in [12], we obtain

$$
[\bar{X}]^{\text {vir }}=\sum_{k \neq 0}\left[\bar{X}^{T, k}\right]^{\text {vir }}=\sum_{k \neq 0} e\left(\bar{\pi}_{(k)}^{*} \mathcal{N}_{(-k)}^{\vee}(1)\right) \cap\left[\mathbb{P}_{X} \mathcal{N}_{(k)}\right]^{\text {vir }}
$$

For $\left[\mathbb{P}_{X} \mathcal{N}_{(k)}\right]^{\text {vir }}$, we notice that the obstruction theory of $\mathbb{P}_{X} \mathcal{N}_{(k)}$ is given by $(3.20)$, and the arrow is given by $\bar{\alpha}_{0}$ in (3.18). Thus using (5.3), we obtain

$$
\left[\mathbb{P}_{X} \mathcal{N}_{(k)}\right]^{\text {vir }}=\sum_{1 \leq i \leq r} a_{i} \cdot\left[\mathbb{P}_{X} \mathcal{N}_{(k)} \times_{X} p_{i}\right]
$$

This proves the Lemma.

We now prove our main theorem.

TheOREM 5.2. Let the situation be as in Theorem 1.1. Let $\left[X^{T}\right]^{\mathrm{vir}}=\sum_{i=1}^{r} a_{i}\left[p_{i}\right]$; let $n_{i, j}$ be the dimension of the weight $j$ part of the tangent space $T_{X, p_{i}}$ and $n_{i}=$ $\sum_{j} n_{i, j}$. Then

$$
\operatorname{deg}\left[M_{+}\right]^{\mathrm{vir}}-\operatorname{deg}\left[M_{-}\right]^{\mathrm{vir}}=\sum_{i} a_{i} \cdot\left((-1)^{n_{i}-1} \sum_{j} \frac{n_{i, j}}{j}\right) .
$$

Proof. By Lemma 5.1 and (5.2), it suffices to show that for any closed point $p \in X^{T}$

$$
\sum_{j \neq 0} \operatorname{res}_{t=0} \frac{e\left(\bar{\pi}_{(j)}^{*} \mathcal{N}_{(-j)}^{\vee}(1)\right) \cap\left[\mathbb{P}_{(j)} \times_{Y^{T}} p\right]}{e\left(N^{\text {vir }}\right) c_{1}\left(\mathcal{O}_{\bar{Z}}(-2 \bar{E})\right)}=(-1)^{n-1} \sum_{j \neq 0} \frac{n_{j}}{j},
$$

where $n_{j}$ is the dimension of the weight $j$ part $V_{j}$ of the Zariski tangent space of $X$ at $p$ and $n=\sum_{j \neq 0} n_{j}$.

For this purpose, we may assume $X^{T}$ is a point $p$, and $Y$ be so that $\left.N_{Y^{T} / Y}\right|_{p}$ is the moving part of $T_{p} X$. Let $V=\oplus_{j \neq 0} V_{j}$ with $p$ identified with 0 . Then

$$
e\left(\bar{\pi}_{(j)}^{*} \mathcal{N}_{(-j)}^{\vee}(1)\right) \cap\left[\mathbb{P} \mathcal{N}_{(j)} \times_{Y^{T}} p\right]=e\left(V_{-j}^{\vee}(1)\right) \cap\left[\mathbb{P} V_{j}\right]
$$

on $\mathbb{P} V$ and (5.4) is equivalent to

$$
\sum_{j \neq 0} \int_{\mathbb{P} V_{j}} \frac{t e\left(V_{-j}^{\vee}(1)\right)}{e\left(\left.N^{\operatorname{vir}}\right|_{\mathbb{P} V_{j}}\right) c_{1}\left(\left.\mathcal{O}_{\bar{Z}}(-2 \bar{E})\right|_{\mathbb{P} V_{j}}\right)}=(-1)^{n-1} \sum_{j \neq 0} \frac{n_{j}}{j} .
$$

Let $\zeta \in A^{1}\left(\mathbb{P} V_{j}\right)$ be the generator of $A^{*}\left(\mathbb{P} V_{j}\right)$ satisfying $\int_{\mathbb{P} V_{j}} \zeta^{n_{j}-1}=1$. Then

$$
c_{1}\left(\left.\mathcal{O}_{\bar{Z}}(-2 \bar{E})\right|_{\mathbb{P} V_{j}}\right)=2(-j t+\zeta)
$$

Furthermore, we have

$$
e\left(\left.\pi^{*} \Omega_{Y}(-E)\right|_{\mathbb{P} V_{j}}\right)=\prod_{i \neq 0}(-i t-j t+\zeta)^{n_{i}}, \quad e\left(\left.V_{-j}^{\vee}(1)\right|_{\mathbb{P} V_{j}}\right)=\zeta^{n_{-j}} .
$$

The normal bundle of $\mathbb{P} V_{j}$ has Euler class

$$
(j t-\zeta) \prod_{i \neq j, 0}((i-j) t+\zeta)^{n_{i}}
$$


and thus the Euler class of $N^{\text {vir }}$ is

$$
e\left(\left.N^{\mathrm{vir}}\right|_{\mathbb{P} V_{j}}\right)=(j t-\zeta) \prod_{i \neq j, 0}((i-j) t+\zeta)^{n_{i}} / \prod_{i \neq-j, 0}(-i t-j t+\zeta)^{n_{i}}
$$

Therefore we have

$$
\begin{aligned}
& \sum_{j} \int_{\mathbb{P} V_{j}} \frac{t e\left(V_{-j}^{\vee}(1)\right)}{e\left(\left.N^{\operatorname{vir}}\right|_{\mathbb{P} V_{j}}\right) c_{1}\left(\left.\mathcal{O}_{\bar{Z}}(-2 \bar{E})\right|_{\mathbb{P} V_{j}}\right)}= \\
& =\sum_{j} \int_{\mathbb{P} V_{j}} \frac{t \prod_{i \neq 0}(-i t-j t+\zeta)^{n_{i}}}{2(-j t+\zeta)(j t-\zeta) \prod_{i \neq j, 0}((i-j) t+\zeta)^{n_{i}}} \\
& =-\sum_{j} \int_{\mathbb{P} V_{j}} \frac{t^{n_{j}-1} \prod_{i \neq 0}\left(-i-j+\frac{\zeta}{t}\right)^{n_{i}}}{2\left(j-\frac{\zeta}{t}\right)^{2} \prod_{i \neq 0, j}\left(i-j+\frac{\zeta}{t}\right)^{n_{i}}} \\
& =-\sum_{j} \operatorname{res}_{x=0} \frac{x^{-n_{j}} \prod_{i \neq 0}(-i-j+x)^{n_{i}}}{2(j-x)^{2} \prod_{i \neq 0, j}(i-j+x)^{n_{i}}} \\
& =-\sum_{j} \operatorname{res}_{x=0} \prod_{i \neq 0}\left(\frac{-i-j+x}{i-j+x}\right)^{n_{i}} \frac{1}{2(j-x)^{2}} \\
& =-\sum_{j} \operatorname{res}_{z=-j} \prod_{i \neq 0}\left(\frac{-i+z}{i+z}\right)^{n_{i}} \frac{1}{2 z^{2}} . \\
& =\operatorname{res}_{z=0} \prod_{i \neq 0}\left(\frac{-i+z}{i+z}\right)^{n_{i}} \frac{1}{2 z^{2}}=\left.\frac{1}{2} \frac{d}{d z}\right|_{z=0} \prod_{i \neq 0}\left(\frac{-i+z}{i+z}\right)^{n_{i}} \\
& =(-1)^{n-1} \sum_{j \neq 0} \frac{n_{j}}{j} .
\end{aligned}
$$

This proves the theorem.

For example, if there are only two weight spaces of weights 1 and -1 respectively, then the wall crossing is

$$
(-1)^{n_{+}+n_{--1}} \cdot\left(n_{+}-n_{-}\right) \cdot \operatorname{deg}\left[X^{T}\right]^{\text {vir }}
$$

where $n_{+}$and $n_{-}$are the dimensions of the positive and negative weight spaces respectively, of the moving part of the Zariski tangent space.

In the situation of simple wall crossing (Definition 2.1) in $D^{b}(\operatorname{Coh} S$ ) for $S$ a Calabi-Yau 3-fold, we obtain the wall crossing formula formulated in Corollary 1.3. 
Proof of Corollary 1.3. We use the notation of $\S 2.3$. The automorphism group of a point in $X$ lying over $E_{1} \oplus E_{2}$ with $E_{1} \in M_{1}$ and $E_{2} \in M_{2}$ is $\mathbb{Z}_{\nu}$ with $\nu$ in $(2.3)$. Hence

$$
\left[X^{T}\right]=\frac{1}{\nu}\left[M_{1}\right]^{\mathrm{vir}} \times\left[M_{2}\right]^{\mathrm{vir}} .
$$

From the definition of the $\mathbb{C}^{*}$-action on $X$, the nontrivial weights on the Zariski tangent space are $\frac{1}{\nu}$ on $\operatorname{Ext}^{1}\left(E_{2}, E_{1}\right)$ and $-\frac{1}{\nu}$ on $\operatorname{Ext}^{1}\left(E_{1}, E_{2}\right)$ respectively. In the formula of Theorem 5.2, the weights $\pm \frac{1}{\nu}$ that go in the denominator cancel out the size of the automorphism group $\nu$. Thus we obtain the corollary.

REMARK 5.3. In a subsequent paper, we will generalize our wall crossing formula to the case $\mathcal{M}_{ \pm} \subset[X / G]$ where $G$ is any complex reductive group acting on the semistable part $X$ of a projective scheme.

Appendix A. Simple wall crossing for perfect obstruction theories. In this section, we prove a virtual analogue of a wall crossing formula for $\mathbb{C}^{*}$-flips.

Since $\mathbb{C}^{*}$ acts on $X_{ \pm}$with finite stabilizers, a $\mathbb{C}^{*}$-equivariant perfect obstruction theory of $X$ induces a perfect obstruction theory of $M_{ \pm}$. Let $\left[M_{ \pm}\right]^{\text {vir }}$ be the associated virtual fundamental cycle $[1,13]$.

Definition A.1. Suppose $M_{ \pm} \subset \mathcal{M}$ are proper simple flips. Let $d$ be their virtual dimension and $\alpha \in A_{\mathbb{C}^{*}}^{d}(X)$ be an equivariant cohomology class. Then the wall crossing term of $\alpha$ is defined as

$$
\delta[\alpha]=\alpha_{+} \cdot\left[M_{+}\right]^{\mathrm{vir}}-\alpha_{-} \cdot\left[M_{-}\right]^{\mathrm{vir}}
$$

where $\alpha_{ \pm} \in A^{d}\left(M_{ \pm}\right)$are the classes induced by restricting $\alpha$ to $X_{ \pm}$and applying the isomorphisms $A_{\mathbb{C}^{*}}^{*}\left(X_{ \pm}\right) \cong A^{*}\left(M_{ \pm}\right)$.

TheOREM A.2. Let $M_{ \pm}$be simple flips in the quotient stack $\mathcal{M}=\left[X / \mathbb{C}^{*}\right]$ that has a perfect obstruction theory. Suppose $X$ embeds $\mathbb{C}^{*}$-equivariantly into a smooth DM-stack. Then for $\alpha \in A_{\mathbb{C}^{*}}^{d}(X)$, the wall crossing is given by

$$
\delta[\alpha]=\sum_{i} \operatorname{res}\left(\alpha \cdot \frac{\left[X_{i}\right]^{\mathrm{vir}}}{e\left(N_{i}^{\mathrm{vir}}\right)}\right) \in \mathbb{Q},
$$

where $X_{i}$ are connected components of $X^{\mathbb{C}^{*}}$ and $N_{i}^{\text {vir }}$ are the $\mathbb{C}^{*}$-equivariant virtual normal bundles of $X_{i}$ in $X$.

Here the residue operator res $=\operatorname{res}_{t=0}$ is taken after expanding the right hand side as a Laurent series in $t$, where $t$ is the generator of $A_{\mathbb{C}^{*}}^{*}(p t)$.

Let $t \in A_{\mathbb{C}^{*}}^{1}(p t)$ be the generator of the $H$-equivariant Chow ring $A_{\mathbb{C}^{*}}^{*}(p t)$. Let $\alpha \in A_{T}^{d}(X)$. Let $\alpha_{ \pm} \in A^{d}\left(M_{ \pm}\right)$be the classes induced by restricting $\alpha$ to $X_{ \pm}$and applying the isomorphisms $A_{T}^{*}\left(X_{ \pm}\right) \cong A^{*}\left(M_{ \pm}\right)$. Let $\tilde{\alpha} \in A^{d}(Z)$ be the class induced by pulling back $\alpha$ to $\left(X \times \mathbb{P}^{1}\right)^{s}$ and applying the isomorphism

$$
A_{T}^{*}\left(\left(X \times \mathbb{P}^{1}\right)^{s}\right) \cong A^{*}(Z)
$$

Let $\alpha_{i}$ be the restriction of $\alpha$ to the fixed point component $X_{i}$. Then the restrictions of $t \tilde{\alpha}$ to the fixed point set $M_{ \pm}$and $X_{i}$ coincide with $t \alpha_{ \pm}$and $t \alpha_{i}$.

We apply the virtual localization theorem [8] to obtain the following. 
Lemma A.3. Let $X$ be a DM-stack equipped with a $\mathbb{C}^{*}$-equivariant perfect obstruction theory and $X$ embeds $\mathbb{C}^{*}$-equivariantly into a smooth DM-stack. Then we have

$$
[Z]^{\mathrm{vir}}=\imath_{*} \sum \frac{\left[X_{i}\right]^{\mathrm{vir}}}{e\left(N_{i}^{\mathrm{vir}}\right)}+\left[M_{+}\right]^{\mathrm{vir}} /(-t)+\left[M_{-}\right]^{\mathrm{vir}} / t
$$

Here $X_{i}$ are the connected components of the $T$-fixed locus $X^{T}$ of $X$, and $N_{i}^{\text {vir }}$ denotes the virtual normal bundle of $X_{i}$ in $X$. Note that if $X$ satisfies the virtual localization requirement, so does $Z$ by construction.

We pair the homology class $[Z]^{\text {vir }}$ with $t \tilde{\alpha} \in A_{H}^{d+1}(Z)$ and then take the degree zero part in $t$. Since $[Z]^{\text {vir }} \in A_{d+1}^{H}(Z)$ has only terms of nonnegative degrees in $t$,

$$
\operatorname{deg}\left(t \tilde{\alpha} \cdot[Z]^{\text {vir }}\right)=0
$$

Therefore, upon moving the terms for $M_{ \pm}$to the left hand side, we obtain the desired wall crossing formula:

$$
\delta[\alpha]=\sum_{i} \operatorname{res}\left(\alpha \cdot \frac{\left[X_{i}\right]^{\mathrm{vir}}}{e\left(N_{i}^{\mathrm{vir}}\right)}\right) \in \mathbb{Q} .
$$

This completes the proof of Theorem A.2.

\section{REFERENCES}

[1] K. Behrend and B. Fantechi, The intrinsic normal cone, Invent. Math., 128:1 (1997), pp. 4588 .

[2] K. Behrend, Donaldson-Thomas invariants via microlocal geometry, Ann. of Math. (2), 170:3 (2009), pp. 1307-1338.

[3] H.-L. Chang And J. Li, On weak perfect obstruction theory, in preparation.

[4] I. Dolgachev and Y. Hu, Variation of geometric invariant theory quotients, Inst. Hautes Etudes Sci. Publ. Math., 87 (1998), pp. 5-56.

[5] G. Ellingsrud and L. Göttsche, Variation of moduli spaces and Donaldson invariants under change of polarization, J. Reine Angew. Math., 467 (1995), pp. 1-49.

[6] R. Friedman and Z. QIn, Flips of moduli spaces and transition formulas for Donaldson polynomial invariants of rational surfaces, Comm. Anal. Geom., 3 (1995), no. 1-2, pp. 11-83.

[7] D. Gieseker, On the moduli of vector bundles on an algebraic surface, Ann. of Math. (2), 106:1 (1977), pp. 45-60.

[8] T. Graber and R. Pandharipande, Localization of virtual classes, Invent. Math., 135:2 (1999), pp. $487-518$.

[9] D. Huybrechts And M. Lehn, The geometry of moduli spaces of sheaves, Aspects of Mathematics, E31. Friedr. Vieweg \& Sohn, Braunschweig, 1997.

[10] D. Joyce And Y. Song, A theory of generalized Donaldson-Thomas invariants, Mem. Amer. Math. Soc., 217 (2012).

[11] Y.-H. KIEM AND J. LI, Localizing virtual cycles by cosections, to appear in Jour. Amer. Math. Soc.

[12] B. Kim, A. Kresch, And T. PAntev, Functoriality in intersection theory and a conjecture of Cox, Katz, and Lee, J. Pure Appl. Algebra, 179 (2003), no. 1-2, pp. 127-136.

[13] J. Li AND G. Tian, Virtual moduli cycles and Gromov-Witten invariants of algebraic varieties, J. Amer. Math. Soc., 11:1 (1998), pp. 119-174.

[14] M. Lieblich, Moduli of complexes on a proper morphism, J. Algebraic Geom., 15:1 (2006), pp. $175-206$.

[15] D. Maulik, R. Pandharipande, and R. Thomas, Curves on K3 surfaces and modular forms. With an appendix by A. Pixton, J. Topol., 3:4 (2010), 937996.

[16] M. Maruyama, Moduli of stable sheaves I. and II, J. Math. Kyoto Univ., 17:1 (1977), pp. 91126 and 18:3 (1978), pp. 557-614. 
[17] K. Matsuki And R. Wentworth, Mumford-Thaddeus principle on the moduli space of vector bundles on an algebraic surface, Internat. J. Math., 8:1 (1997), pp. 97-148.

[18] T. Mochizuki, Donaldson type Invariants for Algebraic Surfaces, Lecture Notes in Mathematics, no. 1972, Springer.

[19] R. Pandharipande and R. Thomas, Curve counting via stable pairs in the derived category, Invent. Math., 178:2 (2009), pp. 407-447.

[20] M. Romagny, Group actions on stacks and applications, Michigan Math. J., 53:1 (2005), pp. 209-236.

[21] C. Simpson, Moduli of representations of the fundamental group of a smooth projective variety. I, Inst. Hautes Etudes Sci. Publ. Math., 79 (1994), pp. 47-129.

[22] C. Simpson, The Hodge filtration on nonabelian cohomology, Algebraic geometry - Santa Cruz 1995, pp. 217-281, Proc. Sympos. Pure Math., 62, Part 2, Amer. Math. Soc., Providence, RI, 1997.

[23] M. Thaddeus, Geometric invariant theory and flips, J. Amer. Math. Soc., 9:3 (1996), pp. 691723. 